\title{
Are You Two Just Friends? Emotional and Sexual Infidelity Across Sexual Orientations
}

\author{
Samantha Yim ${ }^{1}$, Nicole Tanzer ${ }^{1}$, Margaret Satchwell ${ }^{1}$, Coty Chen ${ }^{1}$, Juanshu $\mathrm{Wu}^{1}$, Daniel Javidi ${ }^{1}$, Marissa \\ Hensley ${ }^{1}$, Catherine Phan ${ }^{1} \&$ Jarryd Willis ${ }^{1}$ \\ ${ }^{1}$ Department of Psychology, The University of California, San Diego, USA \\ Correspondence: Jarryd Willis, Department of Psychology, The University of California, San Diego, USA. E-mail: \\ Jarryd.Willis@gmail.com
}

Received: May 30, 2022; Accepted: June 15, 2022; Published: July 1, 2022

Conflicts of interest: We have no conflicts of interest to disclose.

Ethics: The UC-San Diego IRB Board approved both studies.

\begin{abstract}
Lesbians are unlikely to ask if it is possible for women and women to be friends. Bisexuals have friends of each sex. It seems that it is primarily heterosexuals who have trouble with sex-of-attraction friendships. This study examined how participants perceived the emotional and sexual infidelity of their partner's relationship with a friend differing across sexuality and biological sex. Our participants consisted of a combined sample across two studies $(n=532)$, participants completed measures of their perceived emotional and sexual infidelity towards 10 controlled behaviors that their partners committed with the partner's friends. The data revealed that participants were more concerned with perceived emotional infidelity with sex(es)-of-attraction friends as a function of participants' sexual orientation, sex, and their lover's sexual orientation. Our evidence shows that when in relationships, people feel most threatened by the friend of the partner who possesses the same biological machinery as them. Furthermore, results suggest that people are also more likely to be threatened by their partner's friend, who may have a mutual attraction towards their partner. The effect of the same biological machinery and the mutual attraction on perceived infidelity is additive. The pattern is seen across heterosexual, homosexual, and bisexual relationships.
\end{abstract}

Keywords: infidelity, relationships, sexuality, friendship

\section{Introduction}

Can two heterosexuals truly have an opposite-sex friendship if one is in a relationship and the other is single? (Note 1) Unfortunately, the concern embedded in this question continues to negatively affect the maintenance of heterosexual opposite-sex friendships once there is a difference in their relationship status. In such a scenario, the heterosexual opposite-sex friendship may enter a devalued state until equilibrium has been restored (e.g., the single friend becomes romantically involved or the romantically involved friend becomes single). One key mediating factor may be how long the two of them were friends prior to one of them entering into a relationship, with the expectation that long-term friends would be protected against any significant degree of devaluation. At the same time, the creation of any new friendship with a single heterosexual of the opposite sex while currently in a romantic relationship is viewed with the most suspicion.

Why? The traditional arguments have been that heterosexual opposite-sex friendships introduce greater attraction and romantic ambiguity than same-sex friendships do (Afifi \& Faulkner, 2000; Bleske-Rechek et al., 2012), which may generate sexual tension that is emotionally taxing to negotiate, suppress, and avoid (O'Meara, 1989), and may engender suspicion/jealousy in romantic partners (Rawlins, 1992). Overall, the primary issue appears to be that attraction, even if incidental in nature, complicates the formation and maintenance of friendships with the sex(es)of-attraction (opposite-sex friends) for heterosexuals. (Note 2)

The aforementioned studies excellently discuss the 'effect' that having opposite-sex friends has on heterosexuals, and a synopsis of literature investigating probable 'causes' suggests that this is primarily an interpersonal barrier that heterosexuals have needlessly created for themselves. Consider that individuals of the same-sex are the sex(es)-of-attraction for lesbians and gay men and that individuals of the opposite-sex are the sex(es)-of-attraction 
for heterosexuals. Research finds that lesbians and gay men have more same-sex friends (their sex(es)-of-attraction) than heterosexuals have opposite-sex friends (their sex(es)-of-attraction), and that heterosexuals have more samesex friends (non-attraction) than lesbians and gay men have opposite-sex friends (non-attraction) (Gillespie et al., 2015). Moreover, if heterosexuals' logic of having a barrier when forming friendships with the sex(es)-ofattraction was followed to its conclusion for bisexuals (attraction to males and females), then bisexuals would be unable to form friendships with almost anyone. Instead, bisexual females report more female friends than both straight men (their sex(es)-of-attraction) and straight women (non-attraction), and more male friends than both straight women (their sex(es)-of-attraction) and lesbians (non-attraction) (Gillespie et al., 2015). In addition, bisexual men report more female friends than straight men (sex(es)-of-attraction), and more male friends than both straight women (sex(es)-of-attraction) and lesbians (non-attraction). This research suggests that the depreciation and suspicion of sex(es)-of-attraction friendships are not a norm of human mate selection. Rather, it is simply (and sadly) a heteronorm.

Gender homophily in friendships, however, is normative, as individuals across sexual orientation have a greater number of same-sex friendships over time than opposite-sex friendships (Galupo, 2007; Gillespie et al., 2015; Monsour, 2002). Importantly, this norm persists for lesbians and gay men even though their same-sex friend is also their sex(es)-of-attraction (Galupo, 2007; Gillespie et al., 2015). To be clear, heterosexuals are more likely to befriend individuals they are not attracted to whereas lesbians and gay men are more likely to befriend individuals for whom they may feel sexual attraction. Thus, while it is possible to explain why heterosexuals seem perplexed by $\operatorname{sex}(\mathrm{es})$-of-attraction friendships, it does not validate the legitimacy of their apprehension.

An evolutionary explanation for heterosexuals' discomfort with sex(es)-of-attraction friendships may be that there is a conception risk in heterosexual opposite-sex friendships that does not exist in same-sex friendships (i.e., if a lesbian sleeps with another woman or a gay man with another man it is impossible for pregnancy to result in either scenario). The risk of a child as a result of a possible friendship may be enough of a deterrent for heterosexuals to avoid having many opposite-sex friends. It is noteworthy that straight and gay men have more female friends than straight women and lesbians have male friends, gay men have more female friends than straight men, and lesbians have more male friends than straight women (Gillespie et al., 2015). Such a pattern is consistent with evolutionary predictions as straight females are going to be more cautious/selective in forming close bonds with straight men than with gay men (assumed platonic dyad) and more cautious/selective in forming close bonds with straight men than lesbians would be with straight men (assumed platonic dyad). However, upon observing that bisexual women report having more male friends than straight females, the conception risk seems less substantial.

Taken together, heterosexuals' interpersonal barrier towards friendships with their sex(es)-of-attraction is embarrassingly disproportionate compared to other sexual orientations. Rather than taken to be an unavoidable, even if incidental, vestigial attribute of ancestral sexual selection processes, the underlying motives for heterosexuals' derogation of such friendships should be theoretically prosecuted to the point that it is no longer accepted as a norm. Moreover, to the degree that these polar-opposite reactions to sex(es)-of-attraction friendships across sexual orientation are effectively chronically accessible, implicit, and require significant self-regulatory effort to overcome, research should exhaustively consider the possibility that lesbians, gay men, and bisexuals have evolved sexual selection mechanisms that are orthogonal to those that exist for heterosexuals. Clearly, issues with sex(es)-of-attraction friendships is not a mechanism shared across sexual orientation, and its absence potentially confers an adaptive advantage among LGB individuals while its presence potentially confers a disadvantage for heterosexuals. There is no reason to assume, as if by default, that sexual selection behaviors of heterosexuals are inherently more advantageous or adaptive - especially since bisexuals do, in fact, regularly raise children with one of their sexes-of-attraction (Badgett et al., 2021). In this scenario, we see one of the theoretical benefits of including bisexuals when considering evolutionary motivations, as bisexuals turn sex(es)-of-attraction into a constant.

That discussion is beyond the scope of the current article. Rather, we consider a factor for resistance to sex(es)-ofattraction friendships which was not considered in the aforementioned lines of research: actors' differential emotional and sexual infidelity concerns across sexual orientation based on the sexual orientation of their romantic partners' friends.

\section{Jealousy}

Social scientists have generally agreed with the assertion by Daly et al. (1982) that jealousy is an emotional "state that is aroused by a perceived threat to a valued relationship or position and motivates behavior aimed at countering the threat." Moreover, Solomon (2000) argued that jealousy also involves the threat of social humiliation resulting from the real (or imaginary) actions of both one's lover and the perpetrator (or imaginary 
perpetrator). Thus, it should come as no surprise that infidelity has been cited as the most common reason for divorce around the world (Betzig, 1989).

According to Buss (1992), men experience more jealousy when it involves sexual rather than emotional infidelity, and women experience more jealousy when it involves emotional rather than sexual infidelity. This gender difference is believed to result from men's desire to establish certainty in their paternity of any offspring produced within a monogamous romantic investiture, and thereby guarantee that any provisions offered to the offspring would be in the service of supporting the development of his genetic material (Buss, 1992; Eagly \& Wood, 1999; McDonald, 2010; Miller \& Maner, 2009; Parker et al., 2022; Rudman \& Glick, 2008). This gender difference is also influenced by women's desire to establish certainty in the emotional investment and the receipt of resource requisitions on behalf of the offspring (Buss, 1992; Eagly \& Wood, 1999; Edlund \& Sagarin, 2009; Lishner et al., 2008; Parker et al., 2022; Rudman \& Glick, 2008). In short, both men and women experience jealousy in their romantic relationships when something of great evolutionary value is at risk: for men, the cumulative cost of an $18+$ year investment of resources in unrelated offspring (in which case both the infidelity of the partner and nonrelation of the child is unknown to him); for women, having $100 \%$ maternity certainty means she would have to raise the child regardless of the loyalty of the child's father. Thus, those whose jealousy arose from valid concerns and who took action to preserve the integrity of their relationship had greater success in reproduction (Miller \& Maner, 2009).

According to Bringle (1991), jealousy emerges due to the interaction between their partners and specific others, and this jealousy increases in intensity when the characteristics of the other are valued by the individual's partner. In accord with SEM theory, greater jealousy was reported when the domain of a rival's achievements was also a domain of high self-relevance to the straight women. Straight women, to a greater degree than men, considered the desires of their romantic partners in identifying rivals who evoked jealousy, suggesting sex differences in the impact of relationship partners on domains of self-definition (DeSteno \& Salovey, 1996). One important display of this was found in a Facebook study done on heterosexual males and females (Muise et al., 2014). When viewing a photo of their spouse with a mutual or unknown friend, women reported more jealousy than men in the study. The jealousy responses to photos with mutual or unknown friends also predicted Facebook search times for the spouses. Women spent more time doing Facebook searches on their spouses than men did. Lastly, the greatest triggers of negative emotions in spouses (particularly women), included taking a photo with a physically attractive rival of the spouse or updating a relationship status to a title that is not fully reflective of complete commitment (Muise et al., 2009).

However, many of these studies and findings look exclusively at heterosexual individuals and do not look at differences within sexualities nor the relevance of sexuality amongst the partner's friends. Could this theory apply across gender and sexuality? With regard to emotional infidelity, lesbians, gay men, and straight women all report greater concerns with emotional infidelity, whereas straight men report greater concerns with sexual infidelity (Sheets \& Wolfe, 2001). The question arises: will lesbian, gay, and bisexual individuals' infidelity concerns differ based on the sexual orientations of their partner's friends? For instance, a straight man whose straight female partner is friends with a gay man? Or a bisexual woman whose straight male partner is friends with a straight woman?

It is noteworthy that Buss' (1992) evolutionary perspective regarding the sex difference in response to sexual versus emotional infidelity has been challenged by a social-structural perspective. Harris (2000) describes a plethora of alternative accounts which detail that the gender differences we observe may not be due to different innate specific psychological mechanisms that respond to different infidelity types, but rather that emotional jealousy and sexual jealousy are due to a more general jealousy mechanism and cognitive appraisal. When one perceives there is a rival, they ascertain that someone else is either threatening a valued interpersonal relationship, reducing their self-esteem \& self-image, and/or threatening their current/prospective social status. With an understanding of sex differences in infidelity, our focus shifts to possible differences varying by sexual orientation.

\section{Sexual Orientation}

Most infidelity research has focused on heterosexual relationships in which reproduction and changes in offspring investment would be a potential outcome of infidelity. These evolutionary mechanisms that alert individuals to reproduction-based infidelity concerns no longer apply in instances of same-sex infidelity. Specifically, if a woman's wife commits sexual infidelity with another woman, a woman's husband commits infidelity with another man, or a man's husband commits sexual infidelity with another man, it is impossible for pregnancy to be the outcome. How do infidelity concerns change when reproduction is no longer a possible outcome? 
The differences in distress over sexual versus emotional infidelity seem to be found only in heterosexuals but not amongst gays, lesbians, or bisexuals (Frederick \& Fales, 2016). According to the double shot-hypothesis (DeSteno \& Salovey, 1996; Harris, 2003), sexual and emotional infidelity are not mutually exclusive, and each sex has its own perceived belief on which type of infidelity goes with the other. Socialization has influenced beliefs of concurrent infidelity where women believe men can have sexual relationships with others without emotion, however, if men have emotional relations, then sexual relations are implied (Harris, 2000). Men, however, may think for women to have sexual relations with another they are already emotionally involved (DeSteno \& Salovey, 1996; Harris, 2003). Thus, people who have female partners should be more upset and threatened than people who have male partners by sexual infidelity as it implies more of a possibility of concurring emotional infidelity. People with male partners should be more upset and threatened by emotional infidelity than people with female partners as it implies more of a possibility of concurring sexual infidelity.

In line with the double-shot hypothesis, (Frederick \& Fales, 2016) showed that heterosexual men were more likely than heterosexual women to be upset by sexual infidelity (54 vs. $35 \%$ ) and less likely than heterosexual women to be upset by emotional infidelity (46 vs. $65 \%$ ). However, for bisexual men and women, the level to which they were upset did not differ significantly if they were dating a man or a woman. However, consistent with the doubleshot hypothesis, bisexual men dating men were more likely than bisexual women dating women to be more upset by sexual infidelity. Gay men and lesbian women also did not differ significantly in how upset they were about sexual infidelity, though other research has found evidence consistent with (Harris, 2003) and supporting (Carpenter, 2012) the double-shot hypothesis among gay men and lesbians. No research has considered sexual and emotional infidelity concerns across sexual orientation as a function of the lover's sex and sexual orientation.

The benefit of including lesbians/gays in romantic relationships research is they enable the isolation of the variable biological sex. The benefit of including bisexuals in romantic relationships research is that they can answer mate preference questions that not even lesbian/gay subjects can answer when attempting to cross-validate gender socialization/constructivist, evolutionary, and adaptationist theories that have predominantly focused on heterosexual relationships. For example, a lesbian does not have to worry about sexual infidelity between her lesbian wife and a gay male friend as there is mutual disinterest in that friendship. Similarly, a heterosexual woman does not have to worry about sexual infidelity between her straight husband and a straight a male friend as there is mutual disinterest in that friendship. Mutual disinterest is impossible when the lover in question is bisexual. There may be asymmetric interest (e.g., a bisexual woman may desire a male without knowing that he is gay), but mutual disinterest is functionally impossible. Thus, while LG individuals allow one to isolate the variable of biological sex (something bisexuals in same-sex romantic relationships also provide), bisexuals uniquely allow for attraction to be treated as a mathematical constant.

Carpenter (2012) stated that "research with bisexual samples would provide an interesting test of the double-shot hypothesis given that these individuals would be predicted to respond based on their current partner rather than their own sex" (p. 34). As such, the present investigation focused on how women's and men's emotional and sexual infidelity concerns differ across sexuality (heterosexual, homosexual, and bisexual), as well as the sex and sexuality of their lover's friends (i.e., potential romantic rivals).

Please note that two studies are reported. The first focused on emotional infidelity and the second on both emotional and sexual infidelity. As a result, there are more participants for analyses related to emotional infidelity in the combined dataset. Moreover, we reported the results based on the type of infidelity (emotional then sexual) rather than as study one and study two. Finally, both studies were approved by the IRB board and data collection took place between 2018 and 2020.

\section{Methods: Emotional Infidelity}

\subsection{Participants}

This study contained 532 undergraduate participants who attend a western university $\left(M_{\text {age }}=20.6\right.$; range $=18-50$; 208 males, 324 females). The ethnoracial breakdown of our sample was as follows: East Asian (27.0\%), South Asian Indian (2.0\%), Asian - unspecified (17.4\%), Hispanic (21.8\%), White (18.8\%), Multiracials (7.8\%), Black (2.6\%), Middle Eastern (2.6\%), and the remainder chose Other or declined to answer. The sexuality composition of our sample was Straight (61.7\%), Bisexual (28.5\%), and Homosexual (9.8\%). Participants were recruited via the SONA Psychology subject pool and given class credit for participation after completion (Sona Systems, https://www.sona-systems.com). See Table 1. 


\subsection{Procedures}

After consenting to participate in the study, participants began the survey by filling out demographic questions and then went on to complete assessment questions regarding their perceived unfaithful behavior between their significant others and their partner's friends. The survey lasted approximately 50 minutes and prior to receiving the survey packet, each participant filled out an informed consent form. The survey was administered in a well-lit, quiet room. Students were debriefed upon completion of the survey.

\subsection{Measures}

We measured participants' perceived degrees of emotional infidelity based on statements including specific behaviors with specific friends of their partners who vary by sex and sexuality. As infidelity can be interpreted in many ways by individuals, we were specific in the behaviors that we used to ensure no forms of sexual behavior were included. They rated differing acts of emotional infidelity using a seven-point Likert Scale, ' 1 ' indicating the act is "not at all" perceived as emotional infidelity and ' 7 ' indicating the act is "absolutely" perceived as emotional infidelity. Particularly, participants' responses indicated how much they believed a particular act with a particular person constitutes infidelity, for example, their partner "Sharing feelings or having emotional intimacy with a straight friend of the opposite sex" and so forth.

\section{Results: Emotional Infidelity}

\subsection{Straight Men Dating Straight Women}

A repeated measures ANOVA with emotional infidelity friendship type (emotional intimacy with a straight crosssex friend vs. straight same-sex friend vs. gay cross-sex friend vs. gay same-sex friend) as the within-subjects factor, was significant, $F(3,327)=44.47, p<.001, \eta_{p}^{2}=29.0 \%$. As expected, bonferroni post-hoc comparisons revealed that straight males reported they would be more concerned with emotional intimacy involving their straight girlfriends' straight male friends $(M=4.08, S E=.18)$ than with their lesbian friends $(M=2.74, S E=.16)$, gay male friends $(M=2.66, S E=.17)$, or straight female friends $(M=2.38, S E=.17)($ all $p s<.001)$.

\subsection{Straight Men Dating Bisexual Women}

A repeated measures ANOVA with emotional infidelity friendship type (emotional intimacy with a straight crosssex friend vs. straight same-sex friend vs. gay cross-sex friend vs. gay same-sex friend) as the within-subjects factor, was significant, $F(3,69)=5.46, p=.002, \eta_{p}^{2}=19.2 \%$. None of the Bonferroni post-hoc comparisons were significant. The silver lining of this analysis was that the descriptive data reflected the theoretically anticipated pattern: straight men's emotional infidelity concern scores were higher for their bisexual girlfriends' straight male friends $(M=3.88, S E=.37)$ than for her gay male friends $(M=3.17, S E=.38)$ or lesbian friends $(M=3.3, S E$ $=.39$ ). In addition, there was a marginal effect for emotional infidelity concern scores between straight male friends and straight female friends $(M=3.0, S E=.40)$.

\subsection{Straight Women Dating Straight Men}

A repeated measures ANOVA with emotional infidelity friendship type (emotional intimacy with a straight crosssex friend vs. straight same-sex friend vs. gay cross-sex friend vs. gay same-sex friend) as the within-subjects factor, was significant, $F(3,471)=57.88, p<.001, \eta_{p}^{2}=26.9 \%$. As expected, bonferroni post-hoc comparisons revealed that straight females reported they would be more concerned with emotional intimacy involving their straight boyfriends' straight female friends $(M=4.46, S E=.15)$ than with their gay male friends $(M=3.26, S E$ $=.16)$, straight male friends $(M=3.02, S E=.17)$, or lesbian friends $(M=2.99, S E=.16)($ all $p$ s $<.001)$.

\subsection{Straight Women Dating Bisexual Men}

A repeated measures ANOVA with emotional infidelity friendship type (emotional intimacy with a straight crosssex friend vs. straight same-sex friend vs. gay cross-sex friend vs. gay same-sex friend) as the within-subjects factor, was significant, $F(3,81)=5.85, p=.001, \eta_{p}^{2}=17.8 \%$. Bonferroni post-hoc comparisons revealed partial support for dual-threat concerns for individuals with a bisexual partner. Straight women reported they would be more concerned with emotional infidelity involving their bisexual boyfriends' straight female friends $(M=4.57$, $S E=.42)$ than with their lesbian friends $(M=3.57, S E=.46 ; p=.029)$ or straight male friends $(M=3.46, S E=.43$; $p=.019)$. We view these results as only partial support because, even though the comparison between emotional infidelity concerns for their boyfriends' straight female friends and gay male friends $(M=4.11, S E=.35)$ was not significant (supporting our dual-threat hypothesis), infidelity concerns for gay male friends did not differ significantly from lesbian friends or straight male friends. 


\subsection{Gay Men Dating Gay Men}

A repeated measures ANOVA with emotional infidelity friendship type (emotional intimacy with a straight crosssex friend vs. straight same-sex friend vs. gay cross-sex friend vs. gay same-sex friend) as the within-subjects factor, was significant, $F(3,54)=12.49, p<.001, \eta_{p}^{2}=41 \%$. As expected, bonferroni post-hoc comparisons revealed that gay men reported they would be more concerned with emotional infidelity involving their gay boyfriends' gay male friends $(M=4.58, S E=.41)$ than with their straight female friends $(M=2.63 ; S E=.43 ; p$ $=.001)$, their lesbian friends $(M=2.53, S E=.57 ; p=.001)$, or straight male friends $(M=3.21, S E=.45 ; p=.002)$.

\subsection{Gay Men Dating Bisexual Men}

A repeated measures ANOVA with emotional infidelity friendship type (emotional intimacy with a straight crosssex friend vs. straight same-sex friend vs. gay cross-sex friend vs. gay same-sex friend) as the within-subjects factor, was significant, $F(3,48)=12.81, p<.001, \eta_{p}^{2}=44.5 \%$. Bonferroni post-hoc comparisons revealed partial support for dual-threat concerns for individuals with a bisexual partner. Gay men reported they would be more concerned with emotional infidelity involving their bisexual boyfriends' gay male friends $(M=4.77, S E=.50)$ than with their lesbian friends $(M=2.59, S E=.45 ; p=.002)$, straight female friends $(M=2.94, S E=.54 ; p=.003)$, or straight male friends $(M=2.88, S E=.54 ; p=.003)$. We view these results as only partial support because, even though gay male friends arose as a clear infidelity threat, straight female friends did not. This may be related to research indicating that straight men are more open to dating bisexual women than straight women are to dating bisexual men. Gay males may be aware of this and thus discount the threat that a straight woman may pose.

\subsection{Bisexual Women Dating Straight Men}

A repeated measures ANOVA with emotional infidelity friendship type (emotional intimacy with a straight crosssex friend vs. straight same-sex friend vs. gay cross-sex friend vs. gay same-sex friend) as the within-subjects factor, was significant, $F(3,204)=27.08, p<.001, \eta_{v}^{2}=28.5 \%$. As expected, Bonferroni post-hoc comparisons revealed that bisexual women reported they would be more concerned with emotional intimacy involving their straight boyfriends' straight female friends $(M=4.03, S E=.26)$ than with their gay male friends $(M=3.54, S E$ $=.25 ; p=.02)$, lesbian friends $(M=2.77, S E=.23 ; p<.001)$, or straight male friends $(M=2.74, S E=.24 ; p$ $<.001)$. Interestingly, bisexual women's emotional infidelity concerns for their straight boyfriends' gay male friends were also significantly greater than for lesbian and straight male friends (both $p \mathrm{~s}<.001$ ). Given that the male partners in question are straight, bisexual women's indication of a dual-threat concern reflects their own sexual orientation as bisexual rather than their boyfriends' orientation as straight. This will be explored further in the discussion.

\subsection{Bisexual Women Dating Bisexual Men}

A repeated measures ANOVA with emotional infidelity friendship type (emotional intimacy with a straight crosssex friend vs. straight same-sex friend vs. gay cross-sex friend vs. gay same-sex friend) as the within-subjects factor, was significant, $F(3,60)=4.52, p=.006, \eta_{p}^{2}=18.4 \%$. Contrary to expectations, none of the bonferroni post-hoc comparisons were significant. There was, however, a marginal effect for infidelity concerns regarding their bisexual boyfriends' straight female friends $(M=3.20, S E=.44)$ compared to his straight male friends $(M=$ $2.52, S E=.44 ; p=.074)$. Concerns regarding their gay male friends $(M=3.10, S E=.44)$ and lesbian friends $(M$ $=2.76, S E=.44$ ) descriptively fit expectations of a dual-threat concern for individuals with a bisexual partner.

\subsection{Bisexual Women Dating Bisexual Women}

A repeated measures ANOVA with emotional infidelity friendship type (emotional intimacy with a straight crosssex friend vs. straight same-sex friend vs. gay cross-sex friend vs. gay same-sex friend) as the within-subjects factor, was significant, $F(3,63)=5.68, p=.002, \eta_{p}^{2}=21.3 \%$. Bonferroni post-hoc comparisons revealed partial support for dual-threat concerns for individuals with a bisexual partner. Bisexual women reported they would be more concerned with emotional intimacy involving their bisexual girlfriends' lesbian friends $(M=4.23, S E=.42)$ than with their gay male friends $(M=3.05, S E=.43 ; p=.007)$ or straight female friends $(M=3.46, S E=.42 ; p$ $=.037$ ). We view these results as only partial support because the comparison between emotional infidelity concerns for their girlfriends' lesbian friends and straight male friends $(M=3.55, S E=.41)$ does not fit expectations.

\subsection{Bisexual Women Dating Lesbians}

A repeated measures ANOVA with emotional infidelity friendship type (emotional intimacy with a straight crosssex friend vs. straight same-sex friend vs. gay cross-sex friend vs. gay same-sex friend) as the within-subjects factor, was significant, $F(3,21)=6.32, p=.003, \eta_{p}^{2}=47.4 \%$. Bonferroni post-hoc comparisons revealed that bisexual women would be more concerned with emotional infidelity involving their lesbian girlfriends' lesbian 
friends $(M=5.03, S E=.68)$ than with their girlfriends' gay male friends $(M=2.38, S E=.71 ; p=.045)$. Though no other comparison was significant, the descriptive data reflected the theoretically anticipated pattern: bisexual women's concern scores for emotional infidelity involving their lesbian girlfriends' lesbian friends was descriptively higher than scores for straight male friends $(M=3.0, S E=.66)$ and straight female friends $(M=3.38$, $S E=.82$ ).

\subsection{Bisexual Men Dating Straight Women}

A repeated measures ANOVA with emotional infidelity friendship type (emotional intimacy with a straight crosssex friend vs. straight same-sex friend vs. gay cross-sex friend vs. gay same-sex friend) as the within-subjects factor, was significant, $F(3,42)=3.93, p=.015, \eta_{p}^{2}=21.9 \%$. Contrary to expectations, none of the Bonferroni post-hoc comparisons were significant. The silver lining of this analysis was that the descriptive data reflected the theoretically anticipated pattern: bisexual men reported they would be more concerned with emotional infidelity involving their straight girlfriends' straight male friends $(M=4.00, S E=.47)$ than with their lesbian friends $(M=$ $3.67, S E=.49)$, gay male friends $(M=2.93, S E=.56)$, or straight female friends $(M=3.13, S E=.54)$. Once again, it appears that bisexuals assumed a dual-threat of infidelity from their monosexual partner.

Table 1. Participants' Rating of Emotional Infidelity Based on The Sex and Sexuality of Their Lover's Friend

\begin{tabular}{|c|c|c|c|c|c|c|c|c|c|c|c|c|}
\hline & \multirow[b]{2}{*}{ Mean } & \multicolumn{2}{|c|}{$\begin{array}{l}\text { Straight } \\
\text { Same-Sex } \\
\text { Friend }\end{array}$} & \multirow[b]{2}{*}{ Mean } & \multicolumn{2}{|c|}{$\begin{array}{l}\text { Gay } \\
\text { Same-Sex } \\
\text { Friend }\end{array}$} & \multirow[b]{2}{*}{ Mean } & \multicolumn{2}{|c|}{$\begin{array}{l}\text { Straight } \\
\text { Opposite-Sex } \\
\text { Friend }\end{array}$} & \multirow[b]{2}{*}{ Mean } & \multicolumn{2}{|c|}{$\begin{array}{l}\text { Gay } \\
\text { Opposite- } \\
\text { Sex Friend }\end{array}$} \\
\hline & & $S D$ & $n$ & & $S D$ & $n$ & & $S D$ & $n$ & & $S D$ & $n$ \\
\hline Gay M \& Gay M & 3.21 & 1.96 & 19 & 4.58 & 1.81 & 19 & 2.63 & 1.89 & 19 & 2.53 & 2.04 & 19 \\
\hline Gay M \& & & & & & & & & & & & & \\
\hline Bisexual M & 2.88 & 2.23 & 17 & 4.76 & 2.08 & 17 & 2.94 & 2.22 & 17 & 2.59 & 1.84 & 17 \\
\hline Straight $M \quad \&$ & & & & & & & & & & & & \\
\hline Straight W & 2.38 & 1.74 & 110 & 2.74 & 1.69 & 110 & 4.08 & 1.92 & 110 & 2.65 & 1.79 & 110 \\
\hline Straight $M \& B i$ & & & & & & & & & & & & \\
\hline W & 2.92 & 1.93 & 24 & 3.25 & 1.85 & 24 & 3.88 & 1.83 & 24 & 3.08 & 1.84 & 24 \\
\hline Bi M \& Gay M & 2.67 & 2.12 & 9 & 3.44 & 2.07 & 9 & 3.11 & 2.03 & 9 & 2.22 & 1.92 & 9 \\
\hline Bi M \& Straight & & & & & & & & & & & & \\
\hline W & 3.00 & 2.10 & 16 & 3.50 & 1.97 & 16 & 4.13 & 1.82 & 16 & 2.88 & 2.09 & 16 \\
\hline $\mathrm{Bi} M \& \mathrm{Bi} W$ & 3.50 & 2.14 & 8 & 3.50 & 2.0 & 8 & 4.0 & 2.20 & 8 & 3.38 & 2.13 & 8 \\
\hline Bi M \& Bi M & 2.80 & 2.49 & 5 & 2.80 & 2.49 & 5 & 2.80 & 2.49 & 5 & 2.60 & 2.51 & 5 \\
\hline Lesbian \& & & & & & & & & & & & & \\
\hline Lesbian & 2.46 & 1.85 & 13 & 2.77 & 1.96 & 13 & 2.38 & 1.94 & 13 & 2.23 & 1.88 & 13 \\
\hline Lesbian \& Bi W & 3.40 & 1.82 & 5 & 5.00 & 0.71 & 5 & 4.80 & 1.79 & 5 & 3.00 & 2.12 & 5 \\
\hline Straight W \& & & & & & & & & & & & & \\
\hline Straight M & 3.00 & 2.13 & 157 & 3.26 & 1.99 & 157 & 4.46 & 1.88 & 157 & 2.99 & 1.99 & 157 \\
\hline Straight $\mathrm{W} \& \mathrm{Bi}$ & & & & & & & & & & & & \\
\hline M & 3.46 & 2.19 & 28 & 4.11 & 1.87 & 28 & 4.57 & 1.91 & 28 & 3.57 & 1.67 & 28 \\
\hline Bi W \& Lesbian & 3.58 & 2.19 & 12 & 4.33 & 2.06 & 12 & 2.92 & 1.68 & 12 & 2.50 & 1.78 & 12 \\
\hline Bi W \& Straight & & & & & & & & & & & & \\
\hline M & 2.71 & 1.96 & 70 & 3.50 & 2.08 & 70 & 4.01 & 2.10 & 70 & 2.76 & 1.92 & 70 \\
\hline $\mathrm{Bi} W \& \mathrm{Bi} W$ & 3.45 & 1.95 & 22 & 4.23 & 1.95 & 22 & 3.55 & 1.92 & 22 & 3.05 & 2.04 & 22 \\
\hline $\mathrm{Bi} W \& \mathrm{Bi} M$ & 2.52 & 2.02 & 21 & 3.10 & 2.0 & 21 & 3.19 & 2.02 & 21 & 2.76 & 2.02 & 21 \\
\hline
\end{tabular}

\section{Methods: Sexual Infidelity}

\subsection{Participants}

Participants were recruited via the SONA Psychology subject pool and given class credits for participation after completion (Sona Systems, https://www.sona-systems.com). This study contained 337 undergraduate participants who attend a western university $\left(M_{\text {age }}=20.67\right.$; range $=18-50 ; 149$ males, 188 females. The ethnic breakdown of our sample was as follows: East Asian (43.1\%), South Asian Indian (3.2\%), Hispanic (20.8\%), White (18.5\%), Middle Eastern (4.2\%), Multiracials (8.3\%), and Black (1.9\%). The sexuality composition of our sample was Straight (62.8\%), Bisexual (26.8\%), and Homosexual (10.4\%). The remainder of the participants choose Other, 
Pansexual, or otherwise did not complete the survey; their results were not used in the analysis. (Note 3) See Table 2.

\subsection{Procedures}

The survey was administered online through Qualtrics and students signed up through SONA. After consenting to participate in the study, participants began the survey by filling out demographic questions and then went on to complete assessment questions regarding their perceived unfaithful behavior between their significant others and their partner's friends. The survey lasted approximately 50 minutes and prior to receiving the survey packet, each participant filled out an informed consent form.

\subsection{Measures}

We measured participants' perceived degrees of sexual infidelity based on statements including specific behaviors with specific friends of their partners who vary by sex and sexuality. They rated differing acts of sexual infidelity using a seven-point Likert Scale, '1' indicating the act is "not at all" perceived as sexual infidelity and '7' indicating the act is "absolutely" perceived as sexual infidelity. Particularly, participants' responses indicated how much they believed a certain act with a specific person constitutes infidelity.

\section{Results: Sexual Infidelity}

\subsection{Straight Women Dating Straight Men}

A repeated measures ANOVA with sexual infidelity friendship type (sexual infidelity with a straight cross-sex friend vs. straight same-sex friend vs. gay cross-sex friend vs. gay same-sex friend) as the within-subjects factor, was significant, $F(3,309)=75.22, p<.001, \eta_{p}^{2}=42.2 \%$. As expected, Bonferroni post-hoc comparisons revealed that straight females reported they would be more concerned with sexual infidelity involving their straight boyfriends' straight female friends $(M=5.66, S E=.19)$ than with their gay male friends $(M=3.70, S E=.22)$, straight male friends $(M=2.61, S E=.22)$, or lesbian friends $(M=4.40, S E=.22)$ (all $p \mathrm{~s}<.001)$. It is noteworthy that sleeping with a lesbian friend would evoke more displeasure than sleeping with a gay male friend $(p=.004)$ and sleeping with a gay male friend more displeasure than sleeping with a straight male friend $(p<.001)$. See Figure 1 .

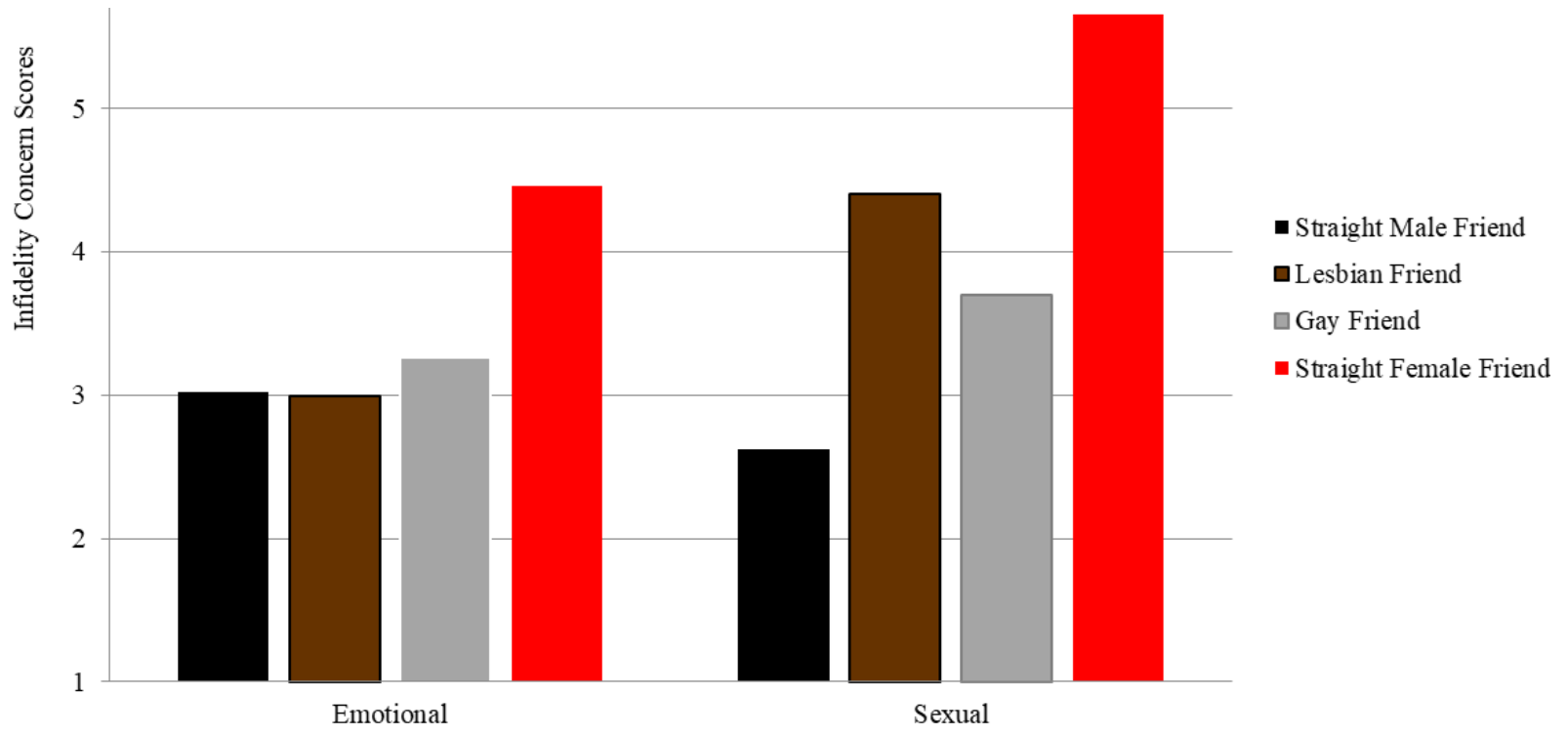

Figure 1. Emotional \& Sexuality Infidelity Concern Scores for Straight Women with Straight Men

\subsection{Straight Men Dating Straight Women}

A repeated measures ANOVA with sexual infidelity friendship type (sexual infidelity with a straight cross-sex friend vs. straight same-sex friend vs. gay cross-sex friend vs. gay same-sex friend) as the within-subjects factor, was significant, $F(3,267)=39.56, p<.001, \eta_{p}^{2}=30.8 \%$. As expected, Bonferroni post-hoc comparisons revealed that straight males reported they would be more concerned with sexual infidelity involving their straight girlfriends' straight male friends $(M=5.18, S E=.23)$ than with their lesbian friends $(M=3.59, S E=.24)$, gay male friends $(M=4.00, S E=.25)$, or straight female friends $(M=2.50, S E=.21)$ (all $p$ s $<.001)$. In addition, they were least 
concerned about her straight same-sex friend compared to other friends (all $p \mathrm{~s}<.001$ ). It is noteworthy that sleeping with her lesbian friend would evoke more displeasure than sleeping next to her straight female friend. See Figure 2 .

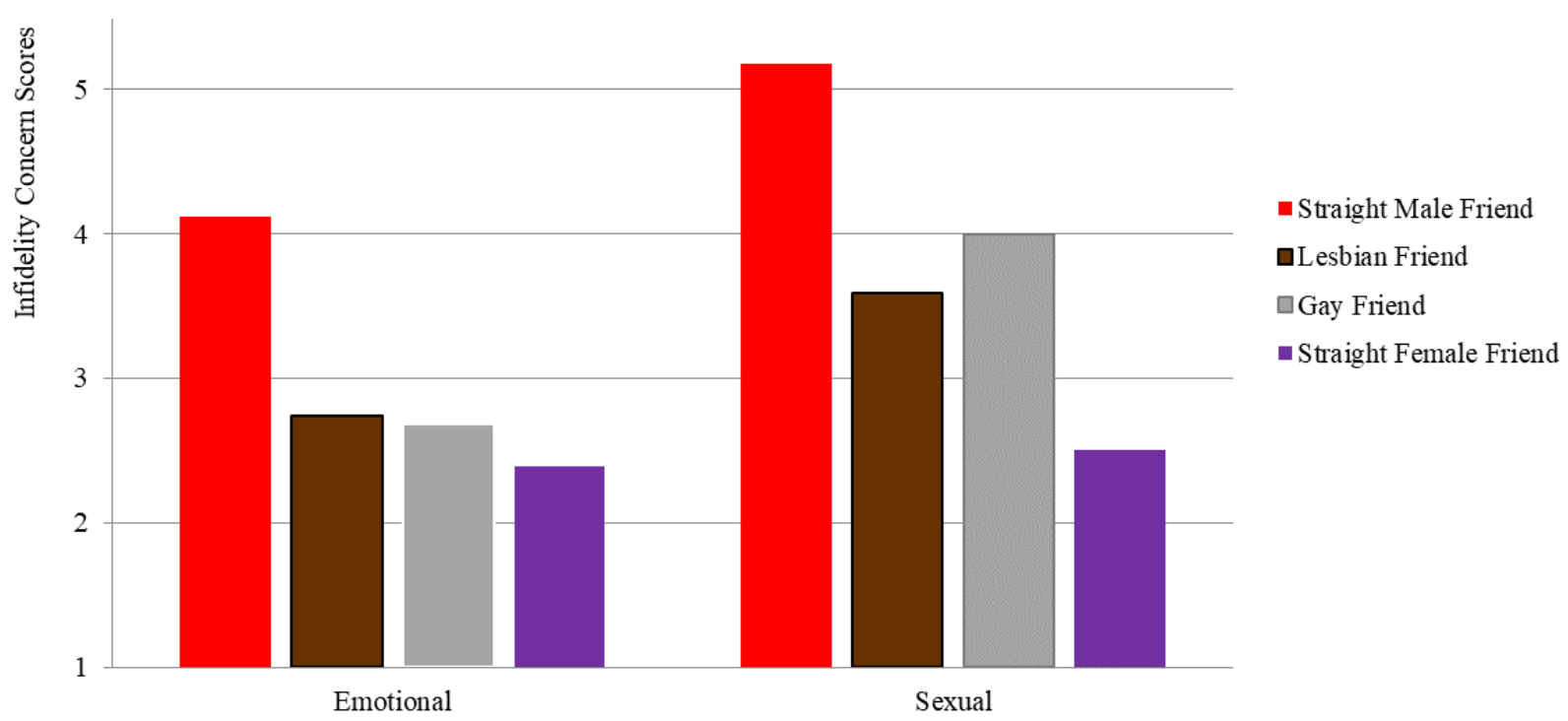

Figure 2. Emotional \& Sexuality Infidelity Concern Scores for Straight Men with Straight Women

\subsection{Bisexual Women Dating Straight Men}

A repeated measures ANOVA with sexual infidelity friendship type (sexual infidelity with a straight cross-sex friend vs. straight same-sex friend vs. gay cross-sex friend vs. gay same-sex friend) as the within-subjects factor, was significant, $F(3,147)=22.45, p<.001, \eta_{p}^{2}=31.4 \%$. Bonferroni post-hoc comparisons revealed that bisexual females reported they would be more concerned with sexual infidelity involving their straight boyfriends' straight female friends $(M=4.86, S E=.42)$ than anyone else (all $p$ s $<.001)$. Moreover, they were less concerned with their boyfriends' straight male friends $(M=2.86, S E=.28)$ than with a lesbian friend $(M=3.64, S E=.31 ; p=.018)$ or gay male friend $(M=3.62, S E=.27 ; p=.003)$. It is noteworthy that straight women dating straight men were significantly more concerned than bisexual women dating straight men about him committing sexual infidelity with a lesbian friend, $F(1,152)=3.94, p=.049, \eta_{p}^{2}=2.5 \%$. See Figures 3 and 4 .

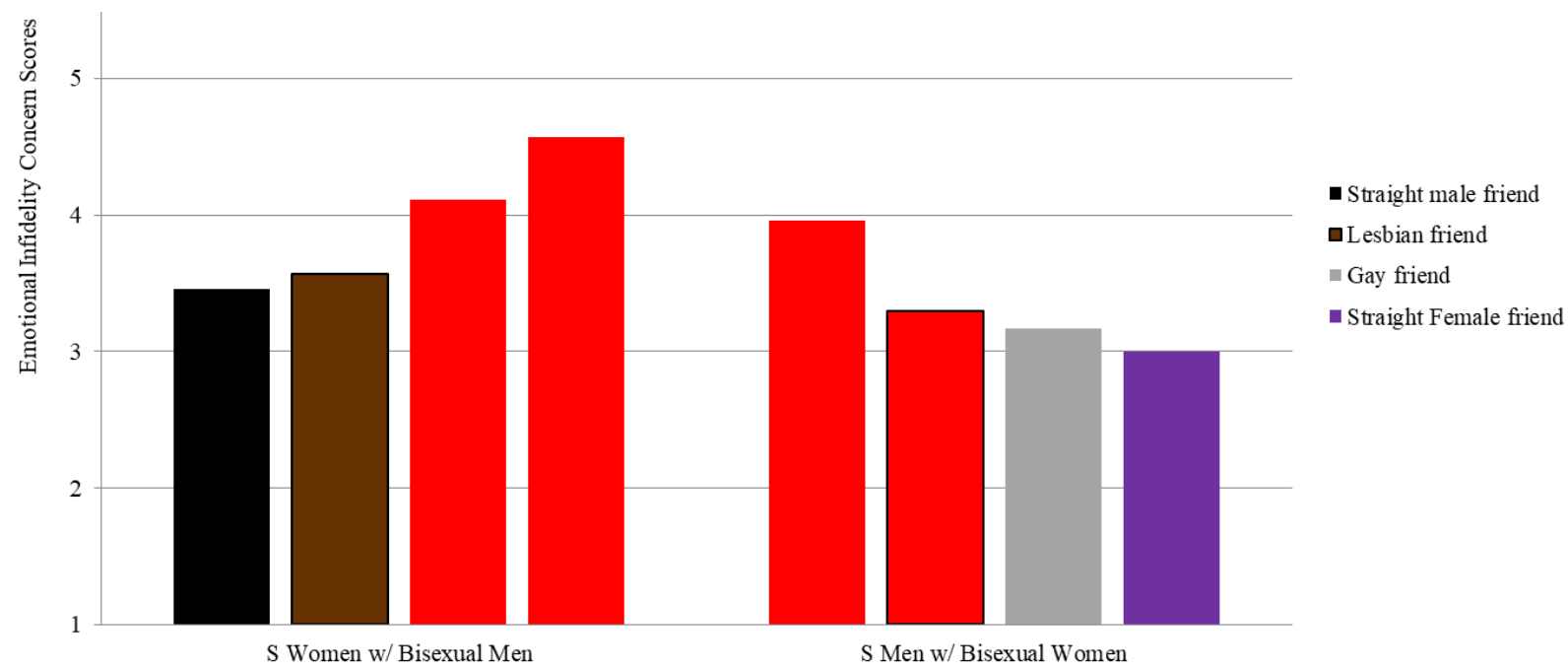

Figure 3. Emotional Infidelity Concern Scores for Straight Women with Bisexual Men and Straight Men with Bisexual Women 


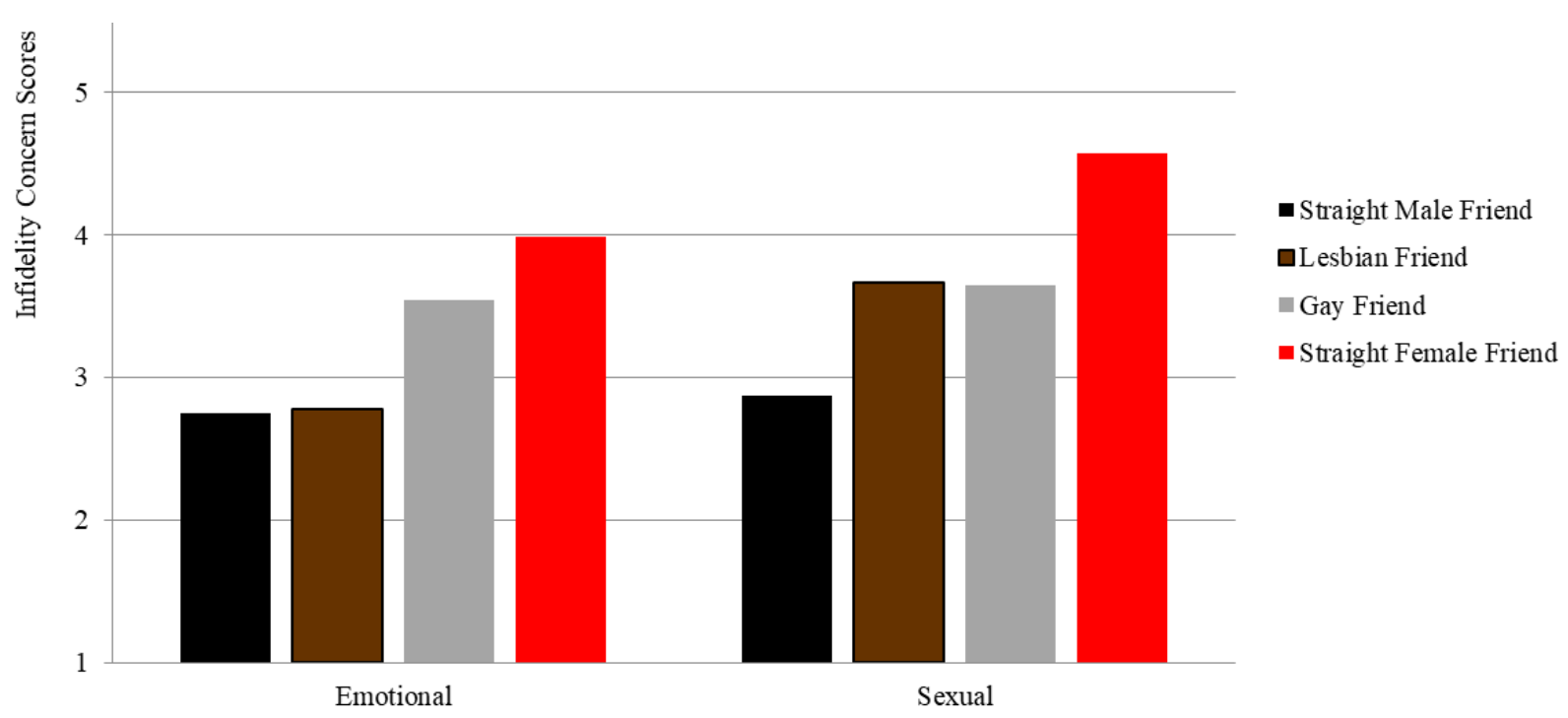

Figure 4. Emotional and Sexuality Infidelity Concern Scores for Bisexual Women with Straight Men

\subsection{Bisexual Women Dating Lesbians}

We would like to preface the following pair of analyses by noting that they were conducted with 7 and 9 participants, respectively. A repeated measures ANOVA with sexual infidelity friendship type (sexual infidelity with a straight cross-sex friend vs. straight same-sex friend vs. gay cross-sex friend vs. gay same-sex friend) as the within-subjects factor, was significant, $F(3,18)=10.71, p<.001, \eta_{p}^{2}=64.1 \%$. Bonferroni post-hoc comparisons revealed that bisexual women reported they would be more concerned with sexual infidelity involving their lesbian girlfriends' lesbian friends $(M=5.0, S E=.66)$ than with their straight male friends $(M=2.14, S E=.26 ; p=.039)$ or gay male friends $(M=1.86, S E=.26 ; p=.049)$. Interestingly, bisexual women's emotional infidelity concerns for their lesbian girlfriends' straight female friends $(M=3.86, S E=.77)$ were descriptively (though not significantly) greater than for male friends. Given that the female partners in question are gay, bisexual women's apparent dual-threat concern reflects their own sexual orientation as bisexual rather than their girlfriends' orientation as lesbian. This will be explored further in the discussion.

\subsection{Lesbians Dating Lesbians}

A repeated-measures ANOVA with sexual infidelity friendship type (sexual infidelity with a straight cross-sex friend vs. straight same-sex friend vs. gay cross-sex friend vs. gay same-sex friend) as the within-subjects factor, was significant, $F(3,24)=3.16, p=.043, \eta_{p}^{2}=28.3 \%$. None of the Bonferroni post-hoc comparisons were significant. The descriptive data reflected the theoretically anticipated pattern, though not as robustly as we expected: lesbians indicated they would be more concerned with sexual infidelity involving their lesbian girlfriends' lesbian friends $(M=2.78, S E=.72)$ than with their straight female friends $(M=2.22 ; S E=.7)$, their gay male friends $(M=2.33, S E=.73)$, or straight male friends $(M=2.44, S E=.71)$.

\subsection{Bisexual Men Dating Straight Women}

A repeated measures ANOVA with sexual infidelity friendship type (sexual infidelity with a straight cross-sex friend vs. straight same-sex friend vs. gay cross-sex friend vs. gay same-sex friend) as the within-subjects factor, was significant, $F(3,21)=3.95, p=.022, \eta_{p}^{2}=36.1 \%$. Once again, none of the Bonferroni post-hoc comparisons were significant, though the descriptive data revealed the expected pattern: bisexual men's concern scores for sexual infidelity involving their straight girlfriends' straight male friends $(M=4.00, S E=.42)$ were higher than with their gay male friends $(M=2.0, S E=.46 ; p=.074)$, lesbian friends $(M=3.0, S E=.57)$, or straight female friends $(M=2.50, S E=.57)$. See Figures 5 and 6 . 


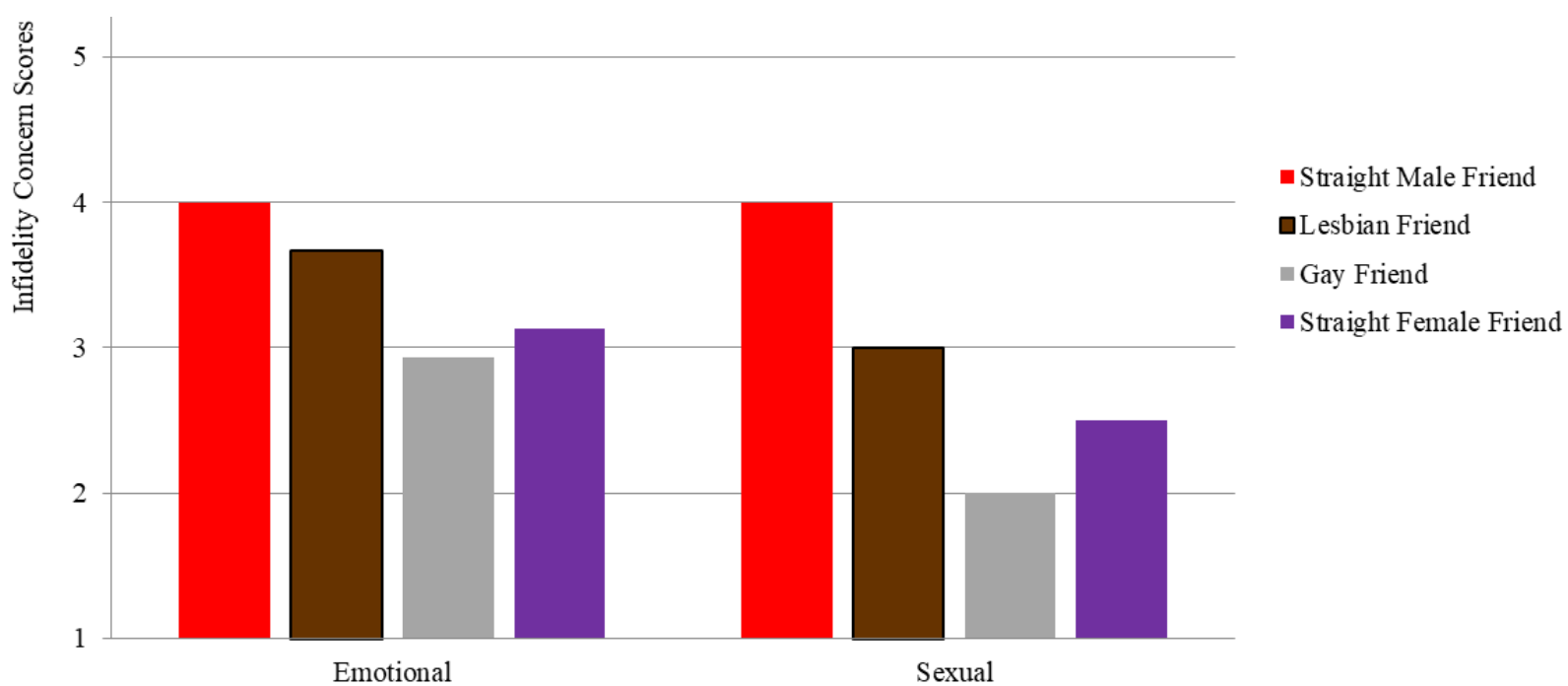

Figure 5. Emotional and Sexuality Infidelity Concern Scores for Bisexual Men with Straight Women

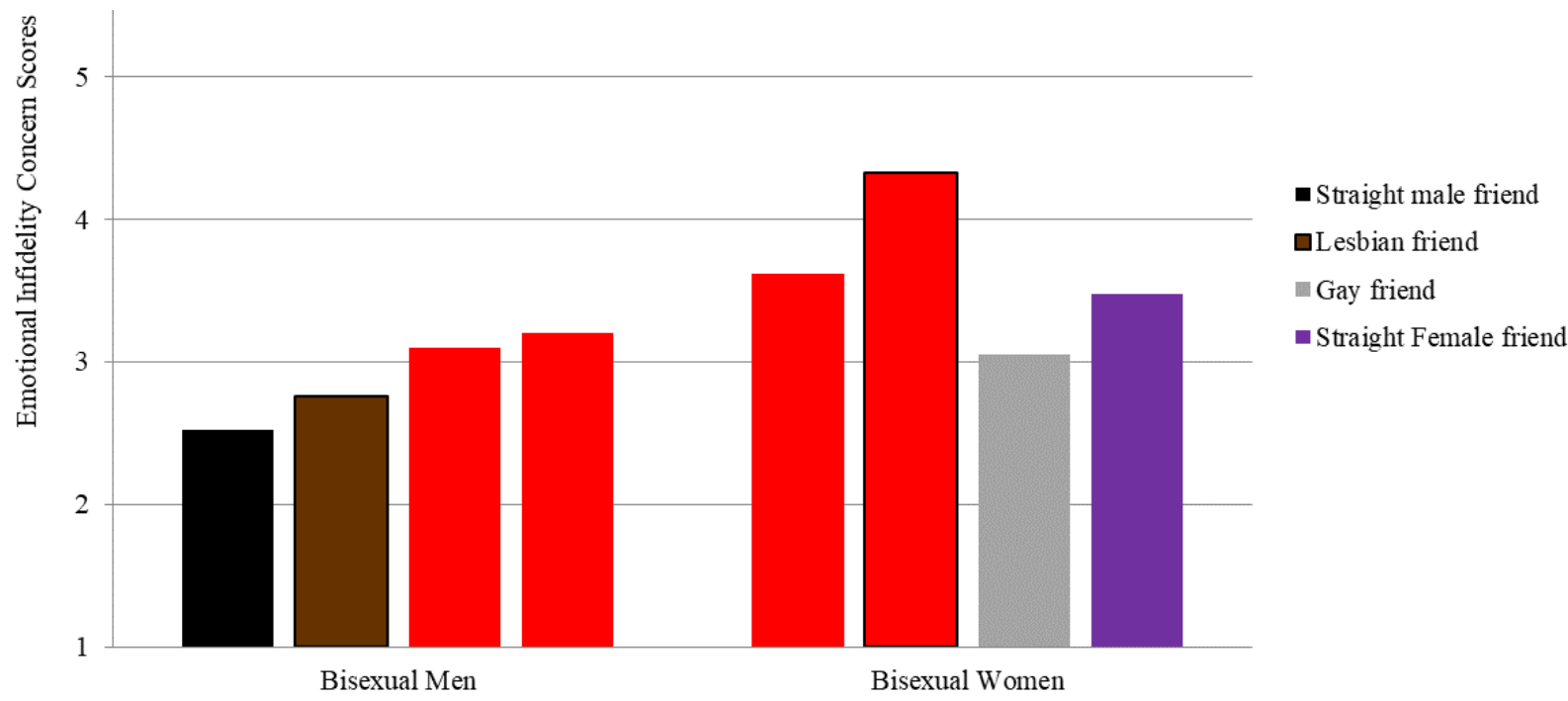

Figure 6. Emotional Infidelity Concern Scores for Bisexual Women with Bisexual Men and Women

\subsection{Gay Men Dating Gay Men}

A repeated-measures ANOVA with sexual infidelity friendship type (sexual infidelity with a straight cross-sex friend vs. straight same-sex friend vs. gay cross-sex friend vs. gay same-sex friend) as the within-subjects factor, was significant, $F(3,36)=18.51, p<.001, \eta_{p}^{2}=60.7 \%$. As expected, Bonferroni post-hoc comparisons revealed that gay men reported they would be more concerned with sexual infidelity involving their gay boyfriends' gay male friends $(M=5.08, S E=.49)$ than with their straight female friends $(M=1.92 ; S E=.33 ; p=.002)$ or their lesbian friends $(M=1.77, S E=.32 ; p=.001)$. They also indicated greater sexual infidelity concerns regarding their gay boyfriends' straight male friends $(M=4.0, S E=.56)$ than with their straight female friends $(p=.048)$ or their lesbian friends $(p=.016)$. There was no significant difference regarding their gay male friends and straight male friends. See Figure 7. 


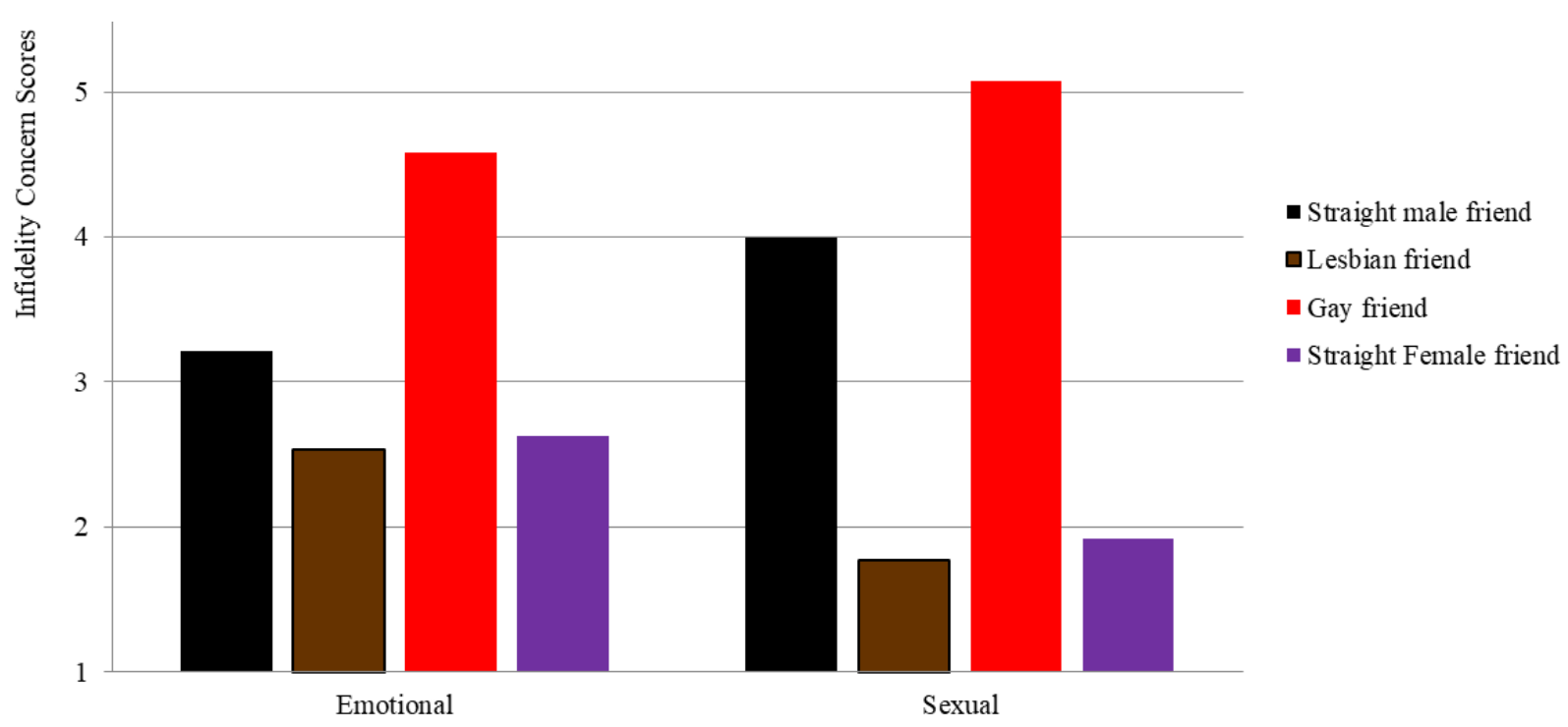

Figure 7. Emotional and Sexuality Infidelity Concern Scores for Gay Men with Gay Men

\subsection{Gay Men Dating Bisexual Men}

A repeated measures ANOVA with sexual infidelity friendship type (sexual infidelity with a straight cross-sex friend vs. straight same-sex friend vs. gay cross-sex friend vs. gay same-sex friend) as the within-subjects factor, was significant, $F(3,27)=7.24, p=.001, \eta_{p}^{2}=44.6 \%$. Bonferroni post-hoc comparisons revealed that gay men reported they would be more concerned with sexual infidelity involving their bisexual boyfriends' gay male friends $(M=4.60, S E=.64)$ than with their lesbian friends $(M=2.20, S E=.57 ; p=.031)$. No differences were found regarding their bisexual boyfriends' straight male friends $(M=3.70, S E=.73)$ or straight female friends $(M=2.90$, $S E=.71)$. See Figure 8.

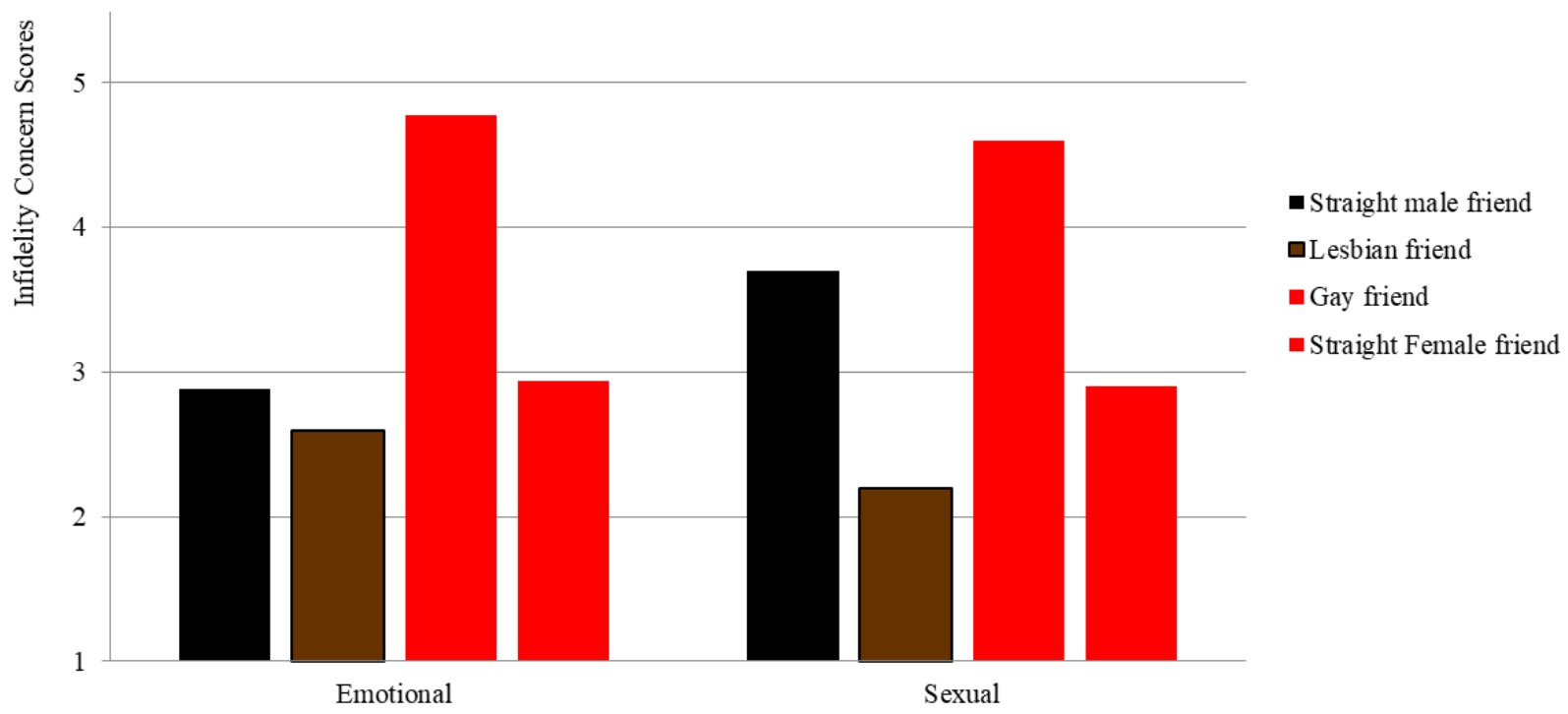

Figure 8. Emotional and Sexuality Infidelity Concern Scores for Gay Men with Bisexual Men 
Table 2. Participants' Ratings of Sexual Infidelity Based on the Sex and Sexuality of Their Lover's Friend

\begin{tabular}{|c|c|c|c|c|c|c|c|c|c|c|c|c|}
\hline & \multirow[b]{2}{*}{ Mean } & $\begin{array}{l}\text { Str } \\
\text { San } \\
\text { Fri }\end{array}$ & $\begin{array}{l}\text { it } \\
\text { Sex }\end{array}$ & & \multicolumn{2}{|c|}{$\begin{array}{l}\text { Gay Same- } \\
\text { Sex Friend }\end{array}$} & & \multicolumn{2}{|c|}{$\begin{array}{l}\text { Opposite-Sex } \\
\text { Friend }\end{array}$} & & \multicolumn{2}{|c|}{$\begin{array}{l}\text { Opposite-Sex } \\
\text { Friend }\end{array}$} \\
\hline & & $S D$ & $n$ & Mean & $S D$ & $n$ & Mean & $S D$ & $n$ & Mean & $S D$ & $n$ \\
\hline Gay M \& & & & & & & & & & & & & \\
\hline Gay M & 4.00 & 2.00 & 13 & 5.08 & 1.75 & 13 & 1.92 & 1.19 & 13 & 1.77 & 1.17 & 13 \\
\hline Gay M \& & & & & & & & & & & & & \\
\hline Bisexual M & 3.70 & 2.31 & 10 & 4.60 & 2.01 & 10 & 2.90 & 2.23 & 10 & 2.20 & 1.81 & 10 \\
\hline Straight $\mathrm{M}$ & & & & & & & & & & & & \\
\hline \& Straight & & & & & & & & & & & & \\
\hline W & 2.50 & 1.94 & 92 & 3.55 & 2.30 & 92 & 5.14 & 2.14 & 92 & 3.97 & 2.37 & 92 \\
\hline Straight $\mathrm{M}$ & & & & & & & & & & & & \\
\hline$\& \mathrm{Bi} \mathrm{W}$ & 3.11 & 2.15 & 9 & 3.89 & 2.32 & 9 & 5.11 & 1.97 & 9 & 3.67 & 2.29 & 9 \\
\hline Bi M \& Gay & & & & & & & & & & & & \\
\hline M & 2.75 & 1.71 & 4 & 4.25 & 2.5 & 4 & 3.50 & 1.92 & 4 & 1.75 & 0.50 & 4 \\
\hline Bi M \& & & & & & & & & & & & & \\
\hline Straight W & 2.78 & 1.72 & 9 & 3.33 & 1.80 & 9 & 4.33 & 1.50 & 9 & 2.44 & 1.81 & 9 \\
\hline $\mathrm{Bi} \mathrm{M} \& \mathrm{Bi}$ & & & & & & & & & & & & \\
\hline $\mathrm{W}$ & 3.14 & 2.04 & 7 & 4.00 & 2.65 & 7 & 4.57 & 2.44 & 7 & 3.29 & 1.89 & 7 \\
\hline $\mathrm{Bi} M \& \mathrm{Bi}$ & & & & & & & & & & & & \\
\hline M & 3.50 & .70 & 2 & 4.00 & 1.41 & 2 & 4.00 & 1.41 & 2 & 2.00 & 1.41 & 2 \\
\hline Lesbian \& & & & & & & & & & & & & \\
\hline Lesbian & 2.22 & 2.11 & 9 & 2.78 & 2.17 & 9 & 2.44 & 2.13 & 9 & 2.33 & 2.18 & 9 \\
\hline Lesbian \& & & & & & & & & & & & & \\
\hline Bi W & 3.00 & 2.00 & 5 & 4.80 & 2.17 & 5 & 4.20 & 2.17 & 5 & 4.00 & 2.35 & 5 \\
\hline Straight W & & & & & & & & & & & & \\
\hline \& Straight & & & & & & & & & & & & \\
\hline M & 2.61 & 2.19 & 104 & 3.70 & 2.23 & 104 & 5.66 & 1.90 & 104 & 4.40 & 2.27 & 104 \\
\hline Bi $\quad$ W \& & & & & & & & & & & & & \\
\hline Lesbian & 3.62 & 2.00 & 8 & 4.63 & 1.92 & 8 & 2.13 & 0.64 & 8 & 1.88 & 0.64 & 8 \\
\hline $\mathrm{Bi} \quad \mathrm{W} \&$ & & & & & & & & & & & & \\
\hline Straight M & 2.86 & 1.97 & 50 & 3.62 & 1.94 & 50 & 4.86 & 1.93 & 50 & 3.64 & 2.16 & 50 \\
\hline $\mathrm{Bi} \mathrm{W} \& \mathrm{Bi}$ & & & & & & & & & & & & \\
\hline W & 3.90 & 2.28 & 10 & 4.60 & 2.22 & 10 & 4.10 & 2.13 & 10 & 3.70 & 2.41 & 10 \\
\hline $\mathrm{Bi} W \& \mathrm{Bi}$ & & & & & & & & & & & & \\
\hline $\mathrm{M}$ & 2.00 & 1.41 & 4 & 2.50 & 1.29 & 4 & 3.75 & 2.50 & 4 & 2.50 & 1.73 & 4 \\
\hline
\end{tabular}

\section{Supplemental Analyses}

\subsection{Demographics Across Both Studies}

Most bisexual women are in relationships with men (Hoang et al., 2011; McLean, 2004; Pond, 2020; Weinberg et al., 1994). In line with this, we found that most bisexual women were dating men (73.8\%) \& most bisexual men were dating women $(68.6 \%), \chi 2(1, N=161)=21.51, p<.001$. Moreover, regarding lover sexuality preferences, bisexual women were more likely to have bisexual girlfriends (73.3\%) and straight boyfriends (76.9\%) whereas bisexual men were more likely to have monosexual boyfriends (54.5\%) and girlfriends $(65.2 \%), \chi 2(3, N=155)=$ $25.15, p<.001$.

The lover sexuality preferences for bisexual women with female lovers ( $73.3 \%$ with bisexual girlfriends) may have less to do with their actual preferences than with research indicating that "lesbians have a more strongly negative impression of bisexual women than gay men do of bisexual men" (Conley et al., 2014). The lover sexuality preferences for bisexual women with male lovers (76.9\% with straight male boyfriends) is consistent with Ess et al. (2022) finding that "bisexual women, unlike any other group, preferred to date men outside of their sexual orientation group, rating heterosexual men over bisexual men" (p. 10). 


\subsection{Dyadic Outness Of Mixed Orientation Couples}

We considered whether or not the outness status of mixed orientation couples (whether or not they were out to parents) would influence the emotional infidelity threat that the heterosexual lover reported regarding a gay samesex rival. We only considered the heterosexual-bisexual dyads in this instance given that bisexuals with a lesbian/gay lover would already be out (as far as romantic rivals are concerned), whereas a bisexual person with a heterosexual lover may be perceived to be straight. As such, the gay same-sex rival may be less concerning for heterosexuals whose bisexual lover is in the closet.

Ultimately, for both straight men dating bisexual women (100\% out) and straight women dating bisexual men (58.3\% out), there was no difference in heterosexuals' infidelity concerns regarding their bisexual lover's gay same-sex friend based on their outness status mixed orientation couples. A limitation in this analysis is that the heterosexual person responded based on the level of outness from their perspective as opposed to answering specifically as to whether or not their bisexual lover had come out to their parents. This will be assessed with more specificity in future research. (Note 4)

\section{Discussion}

\subsection{Summary Of Key Findings}

Although heterosexual relationships and jealousy can be indicative of evolutionary mechanisms, the same phenomena may not be as prevalent among couples of other sexual orientations. The findings of the present study reveal key differences between men and women across sexual orientations regarding concerns about emotional and sexual fidelity as a function of their lover's sex, sexuality, and the sex and sexual orientation of potential romantic rivals. The results from the majority of our findings were consistent with evolutionary motivation in heterosexual relationships and bisexual opposite-sex relationships (reproductive-infidelity), and consistent with the double-shot hypothesis in lesbian, gay, and bisexual same-sex relationships.

\subsection{Monosexual Opposite-Sex Couples}

Consistent with evolutionary theory, heterosexuals were most concerned with the emotional and sexual infidelity of their partners' straight opposite sex friend; the person who poses the greatest threat to their reproductive fitness via the potential loss of resources (Buss, 1992). It is interesting to note that the second-highest friend rated for concern in emotional infidelity for both men and women was the partner's gay friend of the same sex. However, the second-highest friend rated for concern in sexual infidelity for both men and women was the partner's gay friend of the opposite sex (a straight man with a lesbian friend or straight woman with a gay friend). It is possible due to intrasexual competition that they perceive anyone of the same sex as them to be a romantic rival, regardless of the rival's sexual orientation. As mentioned before, straight men and women have fewer opposite-sex friendships, especially when in a mono-orientation relationship, as their partners feel threatened. Since their partner has the ability to be attracted to the opposite sex (regardless of if that individual is not attracted to them gay/lesbian), it causes concern for the person dating them. In contrast, heterosexuals in mixed-orientation relationships with a bisexual lover are more likely to have opposite-sex friendships given the less heteronormative nature of such couples (Jordal, 2011).

\subsection{Monosexual Same-Sex Couples}

As predicted, gay men in relationships with gay men expressed greater sexual and emotional infidelity concerns regarding his boyfriend's gay male friends than for anyone else. Since reproduction is not a factor in homosexual relationships, the concern becomes who is the greatest threat to the stability of their dyad. Moreover, no other friend could possibly pose a threat, since a gay male will have no interest in forming a long-term romantic relationship with straight or gay women, and he would not consider heterosexual male friends to be viable options. It is noteworthy, however, that they ranked their gay boyfriends' straight male friends as the second-highest infidelity threat. We discuss this below in a section focused on several unmerited concerns. Overall, the patterns of gay men's concerns were similar to that of heterosexuals in that the person who is most likely to attract their partner's attention, the friend who is of the same sexuality as the partner, was the friend who garnered the most concern. Unfortunately, our lesbian sample was not large enough to conduct similar analyses; an issue we discuss at greater length in the limitations section.

\subsection{Bisexual Infidelity Concerns}

The most noteworthy findings are those associated with mixed-orientation and bisexual-bisexual couples. Specifically, the interaction of perceived infidelity among bisexuals and monosexuals with bisexual partners. Our original hypothesis was that individuals dating bisexuals would be equally concerned with their partner's opposite 
sex straight and same-sex gay friends. The infidelity concern patterns within several mixed-orientation couples were unquestionably the most puzzling.

Specifically, bisexual participants seemed to impute a dual-threat possibility upon their monosexual partners (e.g., bisexual women with straight men concerned about their gay male friend, bisexual men with straight women concerned about their lesbian friend), and monosexuals (especially males) seemed to appraise same-sex threats as more relevant than their bisexual partner's opposite sex options (straight men with bisexual women only seemed concerned about her straight male friend and discounted her lesbian friend, and gay men dating bisexual men completely discounted his straight female friend). This pattern is potentially an incidental replication and extension of research positing that the actor's point of view plays the dominant role (Howard \& Perilloux, 2017). Thus, this study potentially extends their theory to questions of sexual orientation, even in mixed-orientation couples. Put simply, bisexuals may be more likely to assume a dual-threat of infidelity regardless of their partner's sexuality, and monosexuals may be more likely to assume a single prominent threat even if they are dating someone bisexual.

Alternatively, straight men's greater concern with a bisexual girlfriend's straight male friend is consistent with research indicating that straight men are more concerned about reproductive-infidelity than sexual infidelity with another woman (Confer \& Cloud, 2011; Hughes et al., 2004; Wiederman \& LaMar, 1998). Indeed, "men presented with a same-sex rival were two times less likely" to be distressed about sexual infidelity as straight men whose lover committed sexual infidelity with another man (Voracek et al., 2021). The general explanation for this is that his lover's infidelity with another woman potentially increases his access to another reproductive partner, thus facilitating evolutionary motivations. Another explanation, one that also seeks to explain why straight men are more open than straight women are to bisexual lovers (Gleason et al., 2018; Matsick \& Rubin, 2018), is that a bisexual lover reduces straight men's paternal uncertainty (Apostolou et al., 2017). In the most basic sense, if a bisexual woman is equally as likely to commit sexual infidelity with another woman as she is with a man, whereas a heterosexual woman is only going to commit sexual infidelity with another man, then a lover with same-sex attractions significantly reduces men's concern that they are providing for offspring that they are genetically unrelated to. Given that women are always sure about the maternal certainty (they are sure that they are the mother of the child they gave birth to), relationships with bisexual men would not provide the same adaptive benefit to women. This difference may partially explain why "heterosexual women demonstrate more negative attitudes toward bisexual men than heterosexual men do toward bisexual women" (Gleason et al., 2018, p. 528).

For bisexual women with bisexual men, the globally lower than usual infidelity concern scores may reflect an assumption that bisexual men are not as desired by straight women for them to be a threat, and perhaps are not as desired by gay men. In contrast, bisexual women with bisexual women feel lesbians are a far greater threat than straight men are (given that straight men are roughly equal to straight women, which may just indicate that they are a non-threat, or that bisexual women are projecting themselves onto straight women), and a far greater emotional infidelity threat than gay men are for bisexual women dating bisexual men. These results may be one of the rare cases where someone is perceiving that women would be a greater mate poaching threat than men (in this case gay men) in the corresponding relationship dyad.

A major consistent finding is that bisexuals' sexual infidelity scores for the primary threat are significantly lower $(p<.05)$ than for heterosexuals dating the same person. This is particularly ironic given the misperception that bisexuals are unfaithful (i.e., they are attracted to more people therefore their options to cheat increase). With heterosexuals there is an apparent lack of logical processing when it comes to infidelity concerns, however, bisexuals seem to more accurately take into account the sexuality of their partners' friends. For example, bisexual men perceive the gay male friend as roughly half as concerning as straight men do when dating straight women. Similarly, bisexual women with straight men did not see their lesbian friend as much of a threat in the way straight women dating straight men perceived them. This is not a sex difference with bisexuals. This is a sexuality difference where bisexuals are more accurate at perception and understanding the meaning of sexuality than heterosexuals. Perhaps it reflects heterosexuals' lack of understanding sexuality. Alternatively, bisexuals may be more accurate given that they are more likely to be in mixed-orientation relationships than monosexual individuals (Buxton, 2004; Gates, 2011; Goldberg \& Gartrell, 2014; Legerski et al., 2017; Power et al., 2012).

The finding for bisexual women with a bisexual woman is particularly important, because it suggests that the risk assessment regarding emotional infidelity is not solely concerned about reproduction as an outcome of infidelity. If that was the case, then they would only show a concern for straight men (who her girlfriend could have a child with) \& not lesbian threats (a child would not be a possible outcome of sex). If anything, they are simultaneously concerned with the reproductive threat and a self-referential threat posed by their partner cheating on them with someone possessing the same biological machinery. That is, if her partner leaves her for a man, they have different machinery so there is less of a direct blow to the self. However, if she cheats on her with another woman, someone 
possessing the same machinery, that may lead to a decrease in perceived self-worth. It is not as easy to decouple reproduction and machinery in analyses with monosexual individuals, but bisexuals untie the theoretical knot.

Furthermore, several of the patterns with mixed-orientation couples were partner-sex specific, regardless of individuals' sexual orientation. For example, straight/bisexual men with a bisexual girlfriend were most concerned about her emotional intimacy with a straight male friend (reproduction possible), but lesbians/bisexual women with a bisexual girlfriend were most concerned about her emotional intimacy with a lesbian friend (reproduction not possible). (Note 5) Thus, in these instances, it was the sex of the actor that influenced their infidelity concerns with a bisexual partner, whereas the actor's sexual orientation was rendered irrelevant.

\subsection{Unmerited Concerns}

There were several instances in which participants reported an infidelity threat from an impossible rival. Granted, the unmerited concerns of these rivals never surpassed the functionally plausible rivals, but they should not have differed from the other implausible rivals. For example, the threat that a straight male friend poses to the bond of two gay men is no different from the threat that women (whether gay or straight) pose to their relationship. As such, infidelity threat concerns should be equal for female friends and straight male friends.

Similarly, the threat that lesbian friends pose to the bonds of straight men \& straight women should not differ from the threat posed by her gay male friends or straight female friends. Even if a lesbian friend had an interest in his girlfriend, if his girlfriend is straight then the lesbian friend's interest is irrelevant. Thus, the finding that straight males reported greater sexual infidelity concerns in regard to their straight girlfriends' lesbian friends than her straight female friends suggest they may perceive the lesbian friend is getting something out of it. That is, they may perceive the lesbian friend as extracting some latent relational resource when sleeping next to his straight girlfriend that would not be extracted by gay men or straight women. Similarly, with straight women dating straight men, the same concern arose in which straight women reported greater sexual infidelity concerns with their boyfriend's gay male friends than their straight male friends, indicating they may believe the gay friend is receiving some sort of romantic capital in the friendship.

Alternatively, this may be related to research finding that straight women are more concerned about and treat samesex infidelity with more severity than straight men. Indeed, Franks (2015) stated that "the fact that women became more concerned with sexual infidelity when their partner cheated with a man indicates a negative reaction to a male partner's bisexuality" (p. 348). Indeed, several studies have found that straight women are more distressed by their boyfriend committing sexual infidelity with a man than with a woman (Apostolou, 2019; Confer \& Cloud, 2011; Sagarin et al., 2003; Voracek et al., 2021; Wiederman \& LaMar, 1998). One way to make sense of this is to consider two seemingly unrelated factors: first, the prediction of evolutionary theory that women's concern regarding infidelity is associated with the loss of provisions afforded by the relationship; and second, the greater societal stigma against gay and bisexual men relative to lesbians and bisexual women. The lower societal support for gay and bisexual men may create the perception that a man who leaves a heterosexual-presenting relationship (privileged status) and seeks one with another man must truly want a male lover (given all the societal pressure against him and loss of privileges afforded by heteronormativity). Taken together, a woman who learns of her boyfriend's same-sex affair may feel at greater risk of losing resources of evolutionary value than a woman who learns of her boyfriend's opposite-sex affair, in which case it may have only been a one-time thing and poses no ongoing threat to his desire to continue their relationship.

Oddly, gay men dating bisexual men rated their boyfriend's straight male friends (implausible infidelity risk) as a higher infidelity concern than their straight female friends (plausible infidelity risk). This unmerited concern for their straight male friends over straight female friends may reflect the biphobic beliefs associated with bisexual men. It is possible that when dating a bisexual man, their partners may not believe they are bisexual, but rather in denial of their homosexuality, and therefore perceive them to be interested in men more than women. . Regardless, the concern is unwarranted as straight male friends are an implausible rival, as they would have no romantic interest in the bisexual friend.

\subsection{Limitations}

A key limitation of our study was the lack of lesbian participants we were able to survey. The majority of our participants were collected through SONA, a UCSD psychology tool only accessible to UCSD students where only a small population of students identify as lesbian. An insufficient sample of lesbian participants prohibits a more informative interpretation of lesbians' infidelity concerns in romantic relationships.

Also, our operationalizing of emotional and sexual infidelity contexts was far from exhaustive. We used straightforward scenarios presented to the participants and differentiated them by sexual orientation across their 
partner's friend, which may produce biased results as the participant may have known what we were trying to predict. Further, the scenarios we did use were ones we created ourselves, rather than using an already validated measure of infidelity. Some of the scenarios may not have been as distressing to participants enough to produce the emotions of jealousy that would cause them to have a different response to the array of sexual orientations. Though it is also the case that the distress of various scenarios may differ as a function of sex, sexuality, and gender.

Further, we did not address the feelings or concerns about an individual partner's bisexual friends but focused mainly on their monosexual (straight, gay, and lesbian) friends. By not including bisexuals in the variety of friends assessed as potential rivals, the perception of concern may have been different, or the level of concern based on the different partner's friends could have been lessened or increased. More research with the full spectrum of sexualities for comparison is needed to give more accurate depictions of infidelity concerns amongst sexual orientations and sexes. For instance, asexual individuals generally do not experience sexual attraction or desire for sexual intercourse (Bogaert, 2004; Brotto \& Yule, 2017), and most asexuals are in relationships with non-asexual individuals (Weis et al., 2021). Investigating asexuals' perceptions of and reactions to emotional and sexual infidelity would enrich our understanding of the relevance of courtship, monogamy, commitment, intimacy, affectionate behavior (e.g., holding hands, kissing; Gulledge et al., 2004), and love within relationships in which those attributes are not an outcome of sexual attraction or reducible to sexual intercourse.

Another limitation of our study is that we did not ask women participants whether they were on birth control. Altered levels of hormones have been shown to affect women's preferences, choices, and behaviors. Roberts et al. (2012) found that women using oral contraceptives when they met their partner reported lower levels of sexual satisfaction with him, as well as lower levels of satisfaction with the relationship overall. The effect of birth control may have changed the women's feelings towards their partners or thoughts of their partners while they answered the survey questions making their responses possibly skewed toward less positive responses.

In addition, we did not assess individuals' romantic orientation, which may provide more insight into the patterns found for bisexuals. For instance, while over $90 \%$ of heterosexuals identify as heteromantic and over $80 \%$ of homosexuals identify as homoromantic, only about $60 \%$ of bisexuals identify as biromantic (Clark \& Zimmerman, 2022). Indeed, concordance between sexual orientation and romantic orientation is low for bisexuals and highest for heterosexuals (Clark \& Zimmerman, 2022; Lund et al., 2016). This may be relevant as a bisexual woman who identifies as homoromantic (romantic orientation) heterosexual (sexual orientation) may be more concerned with a straight boyfriend committing sexual infidelity than emotional infidelity, whereas a biromantic bisexual woman may be equally concerned about emotional and sexual infidelity.

Finally, we did not ask participants how fluid they perceived their sexuality to be, or the degree to which they may have reported they were questioning. For example, an individual who participates in a study of this nature at the age of 18 may report that they are straight, and then report that they identify as bisexual at the age of 22 . This could perhaps be ameliorated to a degree by asking participants the age at which they were aware of their sexuality (e.g., at what age did you start to feel like you were straight? Are you sure you are straight? Maybe you have not found the right same-sex lover yet). Similarly, we assessed participants based on their sex and sexuality, but did not collect data regarding their gender. Individuals' gender and expression of their identity may provide additional insights into their perceptions of infidelity (e.g., androgynous lesbian woman with a feminine bisexual girlfriend considering the infidelity risk of a genderfluid romantic rival).

\subsection{Concluding Comments}

Future research into differences in infidelity concerns amongst heterosexual, homosexual, and bisexual relationships should explore the nuances of each type of relationship as it relates to societal norms and socialization. An interesting point of study would be to explore the interaction between unique relationship dynamics and the lack of societal norms for homosexual and bisexual individuals, due to long-standing prejudice and discrimination against them. Since historically they have been underrepresented in mainstream media, discussions, and societal benefits, these dyads have fewer models and pressures of what is considered a "normal" relationship and the facets that go along with it. For instance, bisexuals are more likely than monosexuals to be in polyamorous relationships (Balzarini et al., 2018; Haupert et al., 2017). In addition, some mixed-orientation couples practice gendered monogamy, in which the bisexual spouse may engage in non-monogamy with people of the opposite-sex of their lover (a bisexual man with a wife could be with other men but not women) (Pallotta-Chiarolli, 2014). Our study focused mainly on the out of dyad rival across sexuality and sex, and the differences between them, rather than the degree to which infidelity itself bothered each sexuality. Research addressing the idiosyncratic differences in perceptions of infidelity to each sexuality, as well as non-heteronormative forms of commitment such as 
polyamory and gendered monogamy, should be explored. Further, the determination of the sex differences in infidelity should continually be addressed as there are many confounding theories and reasonings as to why they persist.

\section{Acknowledgements}

We would like to thank Gareth Disler, Laureen Khalil, \& Zhiying Wang for their support at different points of this project.

Link to supplementary figures: https://osf.io/3bfge/?view_only=304650806a9648cb8ae95a37c0374020

\section{References}

Afifi, W. A., \& Faulkner, S. L. (2000). On being just friends': The frequency and impact of sexual activity in crosssex friendships. Journal of Social and Personal Relationships, 17(2), 205-222. https://doi.org/10.1177\%2F0265407500172003

Apostolou, M., Shialos, M., Khalil, M., \& Paschali, M. (2017). The evolution of female same-sex attraction: The male choice hypothesis. Personality and Individual Differences, 116, 372-378. https://doi.org/10.1016/j.paid.2017.05.020

Apostolou, M. (2019). The evolution of same-sex attraction in women: Male tolerance to same-sex infidelity. Journal of Individual Differences, 40(2), 104. https://econtent.hogrefe.com/doi/10.1027/16140001/a000281

Badgett, M. V., Carpenter, C. S., \& Sansone, D. (2021). LGBTQ economics. Journal of Economic Perspectives, 35(2), 141-70. https://doi.org/10.1257/jep.35.2.141

Balzarini, R. N., Shumlich, E. J., Kohut, T., \& Campbell, L. (2018). Sexual attitudes, erotophobia, and sociosexual orientation differ based on relationship orientation. The Journal of Sex Research. https://doi.org/10.1080/00224499.2018.1523360

Betzig, L. (1989). Causes of conjugal dissolution: A cross-cultural study. Current Anthropology, 30(5), 654-676. https://doi.org/10.1086/203798

Bleske-Rechek, A., Somers, E., Micke, C., Erickson, L., Matteson, L., Stocco, C., Schumacher, B., \& Ritchie, L. (2012). Benefit or burden? Attraction in cross-sex friendship. Journal of Social and Personal Relationships, 29(5), 569-596. https://doi.org/10.1177\%2F0265407512443611

Bogaert, A. F. (2004). Asexuality: Prevalence and associated factors in a national probability sample. The Journal of Sex Research, 41(3), 279-287. https://doi.org/10.1080/00224490409552235

Bringle, R. G. (1991). Psychosocial aspects of jealousy: A transactional model. In P. Salovey (Ed.), The psychology of jealousy and envy (pp. 103-131). New York: Guilford. Retrieved from https://link.springer.com/chapter/10.1007/978-1-4615-4773-0_27

Brotto, L. A., \& Yule, M. (2017). Asexuality: Sexual orientation, paraphilia, sexual dysfunction, or none of the above? Archives of Sexual Behavior, 46(3), 619-627. https://doi.org/10.1007/ s10508-016-0802-7

Buss, D. M., Larsen, R. J., Westen, D., \& Semmelroth, J. (1992). Sex differences in jealousy: Evolution, physiology, and psychology. Psychological Science, 3(4), 251-256. https://doi.org/10.1111\%2Fj.14679280.1992.tb00038.x

Buxton, A. P. (2004). Works in progress: How mixed-orientation couples maintain their marriages after the wives come out. Journal of Bisexuality, 4(1-2), 59-82. https://doi.org/10.1300/J159v04n01_06

Carpenter, C. J. (2012). Meta-analyses of sex differences in responses to sexual versus emotional infidelity: Men and women are more similar than different. Psychology of Women Quarterly, 36(1), 25-37. https://doi.org/10.1177/0361684311414537

Clark, A. N., \& Zimmerman, C. (2022). Concordance Between Romantic Orientations and Sexual Attitudes: Comparing Allosexual and Asexual Adults.Archives of Sexual Behavior, 1-11. https://doi.org/10.1007/s10508-021-02194-3

Confer, J. C., \& Cloud, M. D. (2011). Sex differences in response to imagining a partner's heterosexual or

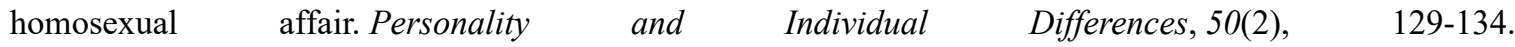
https://doi.org/10.1016/j.paid.2010.09.007

Conley, T. D., Rubin, J. D., Matsick, J. L., Ziegler, A., \& Moors, A. C. (2014). Proposer gender, pleasure, and danger in casual sex offers among bisexual women and men. Journal of Experimental Social Psychology, 55, 
80-88. https://doi.org/10.1016/j.jesp.2014.06.002

Daly, M., Wilson, M., \& Weghorst, S. J. (1982). Male sexual jealousy. Ethology and sociobiology, 3(1), 11-27. https://doi.org/10.1016/0162-3095(82)90027-9

DeSteno, D., Bartlett, M. Y., Braverman, J., \& Salovey, P. (2002). Sex differences in jealousy: Evolutionary mechanism or artifact of measurement?. Journal of personality and social psychology, 83(5), 1103. https://psycnet.apa.org/doi/10.1037/0022-3514.83.5.1103

DeSteno, D. A., \& Salovey, P. (1996). Jealousy and the characteristics of one's rival: A self-evaluation maintenance perspective. Personality and Social Psychology Bulletin, 22(9), 920-932. https://doi.org/10.1177\%2F0146167296229006

Dijkstra, P., Groothof, H. A., Poel, G. A., LAVERMAN, E. T., Schrier, M., \& Buunk, B. P. (2001). Sex differences in the events that elicit jealousy among homosexuals. Personal Relationships, 8(1), 41-54. https://doi.org/10.1111/j.1475-6811.2001.tb00027.x

Eagly, A. H., \& Wood, W. (1999). The origins of sex differences in human behavior: Evolved dispositions versus social roles. American psychologist, 54(6), 408. https://psycnet.apa.org/doi/10.1037/0003-066X.54.6.408

Edlund, J. E., \& Sagarin, B. J. (2009). Sex differences in jealousy: Misinterpretation of nonsignificant results as refuting the theory. Personal Relationships, 16(1), 67-78. https://doi.org/10.1111/j.1475-6811.2009.01210.x

Ess, M., Burke, S. E., \& LaFrance, M. (2022). Gendered Anti-Bisexual Bias: Heterosexual, Bisexual, and Gay/Lesbian People's Willingness to Date Sexual Orientation Ingroup and Outgroup Members. Journal of Homosexuality, 1-18. https://doi.org/10.1080/00918369.2022.2030618

Franks, A. (2015). Franks, A. (2015). Bisexuality revealed through infidelity elicits behavioral immune response in women. Journal of Bisexuality, 15(3), 346-356. https://doi.org/10.1080/15299716.2015.1051609

Frederick, D. A., \& Fales, M. R. (2016). Upset over sexual versus emotional infidelity among gay, lesbian, bisexual, and heterosexual adults. Archives of sexual behavior, 45(1), 175-191. https://doi.org/10.1007/s10508-0140409-9

Galupo, M. P. (2007). Friendship patterns of sexual minority individuals in adulthood. Journal of social and personal relationships, 24(1), 139-151. https://doi.org/10.1177\%2F0265407506070480

Gates, G. J. (2011). How many people are lesbian, gay, bisexual, and transgender? Los Angeles, CA: UCLA School of Law, Williams Institute. https://escholarship.org/uc/item/09h684X2

Gilchrist-Petty, E., \& Bennett, L. K. (2019). Cross-sex best friendships and the experience and expression of jealousy within romantic relationships. Journal of Relationships Research, 10. https://doi.org/10.1017/jrr.2019.16

Gillespie, B. J., Frederick, D. A., Harari, L., \& Grov, C. (2015). Homophily, close friendship, and life satisfaction among gay, lesbian, heterosexual, and bisexual men and women. PLoS One, 10, e0128900. Doi: https://doi.org/10.1371/journal.pone. 0128900

Gleason, N., Vencill, J. A., \& Sprankle, E. (2018). Swipe left on the bi guys: Examining attitudes toward dating and being sexual with bisexual individuals. Journal of Bisexuality, 18(4), 516-534. https://doi.org/10.1080/15299716.2018.1563935

Goldberg, A. E., \& Gartrell, N. K. (2014). LGB-parent families: The current state of the research and directions for the future. Advances in child development and behavior, 46, 57-88. https://doi.org/10.1016/B978-0-12800285-8.00003-0

Gulledge, A. K., Stahmann, R. F., \& Wilson, C. M. (2004). Seven types of nonsexual romantic physical affection among Brigham Young University students. Psychological Reports, 95(2), 609-614. https://doi.org/10.2466/pr0.95.2.609-614

Harris, C. R. (2000). Psychophysiological responses to imagined infidelity: The specific innate modular view of jealousy reconsidered. Journal of personality and social psychology, 78(6), 1082. https://doi.org/10.1037//0022-3514.78.6.1082

Harris, C. R. (2003a). A review of sex differences in sexual jealousy, including self-report data, psychophysiological responses, interpersonal violence, and morbid jealousy. Personality and Social Psychology Review, 7(2), 102-128. https://doi.org/10.1207\%2FS15327957PSPR0702_102-128

Haupert, M. L., Gesselman, A. N., Moors, A. C., Fisher, H. E., \& Garcia, J. R. (2017). Prevalence of experiences 
with consensual nonmonogamous relationships: Findings from two national samples of single Americans. Journal of sex \& marital therapy, 43(5), 424-440. https://doi.org/10.1080/0092623X.2016.1178675

Hoang, M., Holloway, J., \& Mendoza, R. H. (2011). An empirical study into the relationship between bisexual identity congruence, internalized biphobia and infidelity among bisexual women. Journal of Bisexuality, 11(1), 23-38. https://doi.org/10.1080/15299716.2011.545285

Howard, R. M., \& Perilloux, C. (2017). Is mating psychology most closely tied to biological sex or preferred partner's sex?. Personality and Individual Differences, 115, 83-89. https://doi.org/10.1016/j.paid.2016.05.009

Hughes, S. M., Harrison, M. A., \& Gallup, G. G. (2004). Sex differences in mating strategies: Mate guarding, infidelity and multiple concurrent sex partners. Sexualities, Evolution \& Gender, 6(1), 3-13. http://dx.doi.org/10.1080/14616660410001733588

Jordal, C. (2011). "Making it Work": A Grounded Theory of How Mixed Orientation Married Couples Commit, Sexually Identify, and Gender Themselves. (Doctoral dissertation). Virginia Polytechnic Institute and State University, Blacksburg, VA. http://hdl.handle.net/10919/27664

Kleven, H., Landais, C., \& Søgaard, J. E. (2021). Does biology drive child penalties? evidence from biological and adoptive families. American Economic Review: Insights, 3(2), 18398.https://doi.org/10.1257/aeri.20200260

Legerski, E., Harker, A., Jeppsen, C., Armstrong, A., Dehlin, J. P., Troutman, K., \& Galliher, R. V. (2017). Mormon mixed-orientation marriages: Variations in attitudes and experiences by sexual orientation and current relationship status. Journal of GLBT Family Studies, 13(2), 186-209. https://doi.org/10.1080/1550428X.2016.1159163

Lishner, D. A., Nguyen, S., Stocks, E. L., \& Zillmer, E. J. (2008). Are sexual and emotional infidelity equally upsetting to men and women? Making sense of forced-choice responses. Evolutionary Psychology, 6(4). https://doi.org/10.1177\%2F147470490800600412

Lund, E. M., Thomas, K. B., Sias, C. M., \& Bradley, A. R. (2016). Examining concordant and discordant sexual and romantic attraction in American adults: Implications for counselors. Journal of LGBT Issues in Counseling, 10(4), 211-226. https://doi.org/10. 1080/15538605.2016.1233840

McDonald, D. (2010). Swinging: Pushing the boundaries of monogamy? In M. Barker, \& D. Langdridge (Eds.), Understanding non-monogamies (pp. 70-81). New York, NY: Routledge. https://doi.org/10.4324/9780203869802

McLean, K. (2004). Negotiating (Non)Monogamy. Journal of Bisexuality, 4(1-2), 83-97. https://doi.org/10.1300/J159v04n01_07

Miller, S. L., \& Maner, J. K. (2009). Sex differences in response to sexual versus emotional infidelity: The moderating role of individual differences. Personality and individual differences, 46(3), 287-291. https://doi.org/10.1016/j.paid.2008.10.013

Monsour, M. (2002). Women and men as friends: Relationships across the life span in the 21st century. Mahwah, NJ: Lawrence Erlbaum.

Muise, A., Christofides, E., \& Desmarais, S. (2009). More information than you ever wanted: does Facebook bring out the green-eyed monster of jealousy?. Cyberpsychology \& behavior: the impact of the Internet, multimedia and virtual reality on behavior and society, 12(4), 441-444. https://doi.org/10.1089/cpb.2008.0263

Muise, A., Christofides, E., \& Desmarais, S. (2014). 'Creeping' or just information seeking? Gender differences in partner monitoring in response to jealousy on Facebook. Personal Relationships, 21, 35-50. https://doi.org/10.1111/pere.12014

O'Meara, J. D. (1989). Cross-sex friendship: Four basic challenges of an ignored relationship. Sex Roles, 21(7-8), 525-543. http://doi.org/10.1007/BF00289102

Pallotta-Chiarolli, M. (2014). Erasure, exclusion by inclusion, and the absence of intersectionality: Introducing bisexuality in education. Journal of Bisexuality, 14(1), 7-17. https://doi.org/10.1080/15299716.2014.872454

Parker, G., Durante, K. M., Hill, S. E., \& Haselton, M. G. (2022). Why women choose divorce: An evolutionary perspective. Current Opinion in Psychology, 43, 300-306. https://doi.org/10.1016/j.copsyc.2021.07.020

Pond, T. (2021). "It's almost like an ownership of my body": Negotiating Identity and Marginalisation in the Lives 
of Bisexual and Other Plurisexual Women (Doctoral dissertation). Auckland University of Technology. https://openrepository.aut.ac.nz/handle/10292/14024

Power, J. J., Perlesz, A., Brown, R., Schofield, M. J., Pitts, M. K., McNair, R., \& Bickerdike, A. (2012). Bisexual parents and family diversity: Findings from the work, love, play study. Journal of Bisexuality, 12(4), 519538. https://doi.org/10.1080/15299716.2012.729432

Rawlins W.K. (1992). Friendship matters: communication, dialectics, and the life course. Hawthorne, NY: Aldine. https://doi.org/10.4324/9780203791486

Roberts, S. C., Klapilová, K., Little, A. C., Burriss, R. P., Jones, B. C., DeBruine, L. M., Petrie, M., \& Havlíček, J. (2012). Relationship satisfaction and outcome in women who meet their partner while using oral contraception. Proceedings of the Royal Society B: Biological Sciences, 279(1732), 1430-1436. Doi: https://doi.org/10.1098/rspb.2011.1647

Rudman, L., \& Glick, P. (2008). The social psychology of gender: How power and intimacy shape gender relations. New York, NY: Guilford Publications.

Sagarin, B. J., Becker, D. V., Guadagno, R. E., Nicastle, L. D., \& Millevoi, A. (2003). Sex differences (and similarities) in jealousy: The moderating influence of infidelity experience and sexual orientation of the infidelity. Evolution and Human Behavior, 24, 17-23. https://doi.org/10.1016/S1090-5138(02)00106-X

Sheets, V. L. \& Wolfe, M. D. (2001) Sexual jealousy in heterosexuals, lesbians, and gays. Sex Roles, 44, 255-276. https://doi.org/10.1023/A:1010996631863

Solomon, R. C. (2000). The philosophy of emotions. In M. Lewis \& J. M. Haviland-Jones (Eds.), Handbook of emotions (pp. 3-15). New York: Guilford Press.

Sona Systems (n.d.). Sona Systems: Cloud-based Participant Management Software [Computer software]. Sona Systems, Ltd. https://www.sona-systems.com/

Voracek, M., Ward, J., \& Proudfoot, P. (2021). Heterosexual couples and homosexual rivals: Sex differences in romantic jealousy when the rival is the same sex as your partner. https://psyarxiv.com/fbzwk/

Weis, R., Hermann, L., Bauer, C., Miller, T. L., Baba, A., van der Biezen, T., Campos, A., Smiga, J. A., Tomaskovic-Moore, S., Trieu, T. H., Walfrand, A., \& Ziebert, J. (2021). The 2019 asexual community survey summary report. The Ace Community Survey Team https://asexualcensus.wordpress.com/2019asexualcommunity-survey-summary-report/

Weinberg, M. S., Williams, C. J., \& Pryor, D. W. (1994). Dual Attraction: Understanding Bisexuality. New York, NY: Oxford University Press.

Wiederman, M. W., \& LaMar, L. (1998). "Not with Him You Don't!”: Gender and emotional reactions to sexual infidelity during courtship. The Journal of Sex Research, 35, 288-297. https://doi.org/10.1080/00224499809551945

\section{Notes}

Note 1. All content related to sex and gender in this article is focused on ciswomen and cismen.

Note 2. Stated as sexes-of-attraction to avoid giving the presumption that only monosexual attraction exists.

Note 3. It is noteworthy that relationships comprised of a least one bisexual member (calculated by considering both the respondents' sexuality and the sexuality they reported for their lover) were significantly more likely to be interracial than monosexuals' dyads. However, among mono-orientation dyads that were bisexual-bisexual, only opposite sex dyads were interracial whereas same-sex bisexual dyads were equally monoracial and interracial. Overall, heterosexuals' mono-orientation dyads were disproportionately monoracial.

Note 4. There was also a trend in which bisexual women with straight boyfriends who indicated being out seemed less concerned about him committing infidelity with a straight female friend. We hypothesize that a heterosexual lover coming out to their parents as being in a mixed orientation relationship signals commitment to the bisexual lover, thus reducing infidelity concerns. This will be borne out in future research.

Note 5. Though the mixed-orientation lesbian-bisexual same-sex couples $(\mathrm{n}=5)$ and mono-orientation oppositesex bisexual couples $(n=8)$ infidelity concern results were descriptively in the theoretical direction, the sample sizes of those dyads were too small to reach significance. 


\section{Copyrights}

Copyright for this article is retained by the author(s), with first publication rights granted to the journal.

This is an open-access article distributed under the terms and conditions of the Creative Commons Attribution license (http://creativecommons.org/licenses/by/4.0/). 